\title{
REVISIÓN SISTEMÁTICA Y TAXONÓMICA DEL GÉNERO SCELIDOTHERIUM OWEN, 1839 (XENARTHRA, PHYLLOPHAGA, MYLODONTIDAE)
}

\author{
ÁNGEL R. MIÑO BOILINI \\ Centro de Ecología Aplicada del Litoral, CONICET, Ruta 5, km 2,5, 3400, CC 128, Corrientes, Argentina. \\ angelmioboilini@yahoo.com.ar
}

\author{
ALFREDO A. CARLINI \& GUSTAVO J. SCILLATO-YANÉ \\ Departamento Científico Paleontología Vertebrados, Museo de La Plata, CONICET, Paseo del Bosque, s/n, 1900, \\ La Plata, Buenos Aires, Argentina. acarlini@fcnym.unlp.edu.ar, scillato@fcnym.unlp.edu.ar
}

\begin{abstract}
SYSTEMATIC AND TAXONOMIC REVISION OF THE GENUS SCELIDOTHERIUM OWEN, 1839 (XENARTHRA, PHYLLOPHAGA, MYLODONTIDAE). The subfamily Scelidotheriinae (Xenarthra, Phyllophaga, Mylodontidae) has been recorded since the "Friasian" sensu lato (middle Miocene) to Lujanian (late Pleistocene - early Holocene). For the Quaternary four genera: Scelidotherium, Valgipes, Scelidodon and Catonyx are recognized and recorded in Argentina, Bolivia, Chile, Ecuador, Peru and Uruguay. The authors performed a systematic and taxonomic study of the genus Scelidotherium Owen of Argentina. Two valid species are recognized: (i) S. bravardi Lydekker presents records in the provinces of Buenos Aires during the Ensenadan (ca. 1 My - 0.4 My BP; early Pleistocene - middle Pleistocene) and in the Pleistocene of the province of San Luis, and (ii) S. leptocephalum Owen presents records during Bonaerian and Lujanian (ca. 0.4 My - 8.5 ky BP; middle Pleistocene - early Holocene) in the Province of Buenos Aires, in the Lujanian of the provinces of Córdoba, Corrientes, Salta y Formosa; outside argentine territory S. leptocephalum is recorded in the late Pleistocene of Uruguay.
\end{abstract}

Key words: sloths, South America, Quaternary, systematic, taxonomy.

RESUMO - A subfamília Scelidotheriinae (Xenarthra, Phyllophaga, Mylodontidae) se registra desde o "Friasense" s.l. (Mioceno médio) ao Lujanense (Pleistoceno inicial - Holoceno final). Para o Quaternário são reconhecidos quatro gêneros: Scelidotherium, Valgipes, Scelidodon e Catonyx se registram na Argentina, Bolívia, Chile, Equador, Peru e Uruguai. Realiza-se um estudo sistemático e taxonômico do gênero Scelidotherium Owen da Argentina. São reconhecidas duas espécies válidas: (i) S. bravardi Lydekker possui registros nas províncias de Buenos Aires durante o Ensenadense (ca. 1 Ma - 0, 4 Ma AP; Pleistoceno inicial - Pleistoceno médio) e no Pleistoceno de San Luís; e (ii) S. leptocephalum Owen apresenta registros durante o Bonaerense e Lujanenense (0,4 Ma - 8,5 ka AP; Pleistoceno médio - Holoceno inicial) da Província de Buenos Aires; no Lujanense das províncias de Córdoba, Corrientes, Salta e Formosa; fora do território argentino S. leptocephalum se registra no Pleistoceno final do Uruguai.

Palavras-chave: preguiças, América do Sul, Quaternário, sistemática, taxonomia.

\section{INTRODUCCIÓN}

Los Xenarthra incluyen tres grandes grupos: Cingulata, Vermilingua y Phyllophaga (= Tardigrada). A su vez, los Vermilingua y los Phyllophaga integran los Pilosa (Delsuc et al., 2002; Gaudin \& McDonald, 2008). Los Phyllophaga fósiles están constituidos por cuatro clados: Nothrotheriidae Ameghino, 1920, Megatheriidae Gray, 1821, Megalonychidae Gervais, 1855 y Mylodontidae Gill, 1872 (McDonald \& De Iuliis, 2008).

De acuerdo a Pascual et al. (1966), Paula Couto (1979), McDonald \& De Iuliis (2008), los Mylodontidae, están integrados por los Mylodontinae Gill, 1872 y Scelidotheriinae Ameghino, 1904. En particular, los Scelidotheriinae son un grupo sudamericano que, a pesar de tener una distribución geográfica muy amplia, no participaron del Gran Intercambio
Biótico Americano (GIBA) (Hoffstetter, 1952; Scillato-Yané et al., 1995; Woodburne et al., 2006).

La primera revisión comprensiva sobre los escelidoterinos del Neógeno y Cuaternario fue realizada por McDonald (1987) procedentes de Argentina, Bolivia, Brasil, Ecuador y Perú. Según McDonald (1987), McDonald \& Perea (2002) para el cuaternario los Scelidotheriinae están representado por Scelidotherium Owen, 1839 y Catonyx Ameghino, 1891.

Más recientemente y según Miño-Boilini \& Carlini (2009); Miño-Boilini (2012a,b) para el Cuaternario se reconocen los siguientes géneros: Scelidotherium Owen, 1839, Valgipes Gervais, 1873, Scelidodon Ameghino, 1881 y Catonyx Ameghino, 1891 (ver además Pujos, 2000; Cartelle et al., 2009).

Más precisamente para McDonald (1987), McDonald \& Perea (2002) solo mencionan a dos especies dentro de Scelidotherium: (i) Scelidotherium parodii Kraglievich, 
1923, del Piso/Edad Chapadmalalense (Plioceno medio). Sin embargo, Kraglievich (1934) erige el género Scelidotheridium e incluye a Scelidotherium parodii como especie tipo (ver Pascual et al., 1966; y (ii) Scelidotherium leptocephalum, como única especie para los Pisos/Edades EnsenedenseLujanense de la región Pampeana, Argentina, y para el Pleistoceno tardío de Uruguay.

Este es el primero de una serie de tres trabajos sobre la revisión de los escelidoterinos cuaternarios derivados de los estudios de la Tesis Doctoral de Miño-Boilini (2012a); el objetivo de esta primera contribución es realizar la revisión sistemática y taxonómica del género Scelidotherium.

\section{MATERIAL Y MÉTODOS}

\section{Material}

Se estudiaron los especímenes tipo de cada uno de las especies de Scelidotherium, además de los ejemplares referidos, depositados en las colecciones de diferentes instituciones de Argentina (ver abreviaturas institucionales), y de aquellos que no fueron estudiados en revisiones previas efectuadas por otros autores.

Los materiales fueron medidos con un calibre manual "Vernier", con un rango de error de 0,5 mm y un compas de espesor de uso antropológico; las medidas se expresan en milímetros $(\mathrm{mm})$ y fueron incluidas en tablas.

\section{Nomenclatura dentaria}

Es difícil proponer homologías de los dientes de los xenarthros pilosos con los de los otros mamíferos placentarios (McDonald, 2003), por lo tanto se utiliza el término de "caniniforme" y "molariformes" para indicarlos, como fue sugerido entre otros, por Cattoi (en Pascual et al., 1966), Scillato-Yané (1977a,b, 1979, 1980, 1981), y que fue adoptada por numerosos autores (e.g. McDonald \& De Muizon, 2002; Carlini \& Scillato-Yané, 2004; Shockey \& Anaya, 2011). Además, en los Phyllophaga existe una reducción del número total de dientes (McDonald, 2003), generalmente a $5 / 4$, pero que puede llegar a $4 / 3$ (e.g. Octomylodon, ver Scillato-Yané, 1977b).

En este trabajo se sigue la nomenclatura dentaria formalizada por Carlini \& Scillato-Yané (2004), quienes denominaron a los primeros dientes (superior e inferior) como Cf1 y cfl (caniniformes superior e inferior respectivamente) y como Mf1, Mf2, Mf3, Mf4 y mf1, mf2, mf3 a los siguientes (molariformes superiores e inferiores respectivamente), en expresa relación a su homología con las piezas así denominadas en los demás pilosos, y no simplemente atendiendo a la forma de su sección. Por lo tanto, los escelidoterinos presentan la siguiente nomenclatura para las series dentarias: la serie dentaria superior posee cinco dientes (Cf1, Mf1, Mf2, Mf3, Mf4) y 4 (cf1, $\mathrm{mf1}, \mathrm{mf} 2, \mathrm{mf} 3)$ en la serie inferior, pero con la característica que todos ellos están molarizados y sin diastema entre los $\mathrm{Cf} / \mathrm{cf}$ y los $\mathrm{Mf} / \mathrm{mf}$. La formula dentaria es entonces de $5 / 4$ × $2=18$; los caniniformes y molariformes son comprimidos en sentido vestíbulo-lingual. Los molariformes superiores (Mf1-Mf4) en vista oclusal pueden variar su sección desde subtriangulares (e.g. Scelidodon, Catonyx y Valgipes) a subovales (e.g. Scelidotherium).

Para este trabajo los Scelidotheriinae presentan caniniformes aunque, como mencionamos molarizados. Es frecuente en estudios previos de escelidoterinos (e.g. McDonald, 1987; Pujos, 2000; McDonald \& Perea, 2002; Cartelle et al., 2009; Miño-Boilini et al., 2009), los dientes son denominados todos como molariformes, y por lo tanto para ellos la serie dentaria superior sería M1, M2, M3, M4 y M5 y la inferior, $\mathrm{m} 1, \mathrm{~m} 2, \mathrm{~m} 3$ y m4, creemos que la homología debe prevalecer como concepto fundamental previo a cualquier estudio filogenético.

Según Esteban (1996), en general los milodontinos (e.g. Glossotherium, Lestodon) presentan cinco dientes superiores y cuatro dientes inferiores, aunque Mylodon muestra una reducción (ausencia) del primer par de dientes (los $\mathrm{Cf} / \mathrm{cf}$ ). En síntesis, en los milodóntidos la formula dentaria es 5/4; en los Scelidotheriinae la molarización de los Cf1/cf1 y la ausencia de diastema entre el primer diente $(\mathrm{Cf1} / \mathrm{cfl})$ y el segundo (Mf1/mf1) ellos es una apomorfía del clado, carácter que queda oscurecido si considera al primer diente como un molariforme más, atendiendo simplemente a su sección.

\section{Análisis filogenético}

Miño-Boilini (2012a) realizo un análisis filogenético como parte de su Tesis Doctoral con el objetivo de estudiar las relaciones de los escelidoterinos del Cuaternario. Utilizo en total 12 taxones: uno es el grupo externo (Nematherium Ameghino, 1887) y los otros 11 constituyen el grupo interno integrado por los siguientes taxones: Neonematherium flabellatum Ameghino, 1904, Sibyllotherium guenguelianum Scillato-Yané \& Carlini, 1998, Proscelidodon patrius (Ameghino, 1888), P. rothi (Ameghino, 1908), Scelidotheridium parodii Kraglievich, 1934, S. leptocephalum Owen, 1839, S. bravardi Lydekker, 1886 Scelidodon tarijensis (Gervais \& Ameghino, 1880), S. chiliensis (Lydekker, 1886), Valgipes bucklandi (Lund, 1839a) y Catonyx cuvieri (Lund, 1839b). Se utilizaron 25 caracteres morfológicos, de los cuales 17 son cráneodentarios y 8 postcraneanos. Los análisis fueron efectuados con el programa TNT (Tree Analysis Using Parsimony), versión 1.1. (Goloboff et al., 2008) a través de la opción "implicit enumeration". A su vez para calcular el soporte de cada clado aplicó lo opción "Symmetric resampling", realizando 1000 pseudoréplicas de la matriz.

Abreviaturas anatómicas. a, alvéolo; $\mathbf{C f}$, caniniforme superior; cf, caniniforme inferior; cu, cubóides; FEC, faceta ectal calcaneal; FSC, faceta sustentacular calcaneal; Mc, metacarpal; Mf, molariforme superior; mf, molariforme inferior.

Abreviaturas institucionales. AMNH, American Museum of Natural History, New York, Estados Unidos; BM(NH) M, Natural History Museum, Londres, Inglaterra; CTESPZ, Paleozoología Corrientes, Facultad de Ciencias Exactas y Naturales y Agrimensura, Universidad Nacional del Nordeste, Corrientes, Argentina; FMNH P, Field Museum of Natural History, Chicago, Estados Unidos; 
GCF, Grupo Conservacionista de Fósiles, San Pedro, Argentina; MACN, Sección Paleontología Vertebrados, Museo Argentino de Ciencias Naturales "Bernardino Rivadavia", Buenos Aires, Argentina; MCNV, Museo Ciencias Naturales, Valencia, España; MD, Museo Municipal "Carlos Darwin”, Punta Alta, Buenos Aires, Argentina; MFCA, Museo Universitario "Florentino y Carlos Ameghino", Universidad Nacional de Rosario (ex Instituto de Fisiografía y Geología “Alfredo Castellanos), Rosario, Argentina; MLP, División Paleontología Vertebrados, Facultad de Ciencias Naturales y Museo, Universidad Nacional de La Plata, La Plata, Argentina; MMP, Museo Municipal de Ciencias Naturales de Mar del Plata, "Lorenzo Scaglia", Mar del Plata, Argentina; PVE-F, Paleontología Villa Escolar, Formosa, Argentina; PIMUZ A/V, Paleontologisches Institut und Museum der Universitat der Zurich, Zurich, Suiza; RCS, Royal College of Surgeons Museum, Londres, Inglaterra.

Abreviaturas de medidas. Cráneo y mandíbula. AC, ancho bicondilar; AH, ancho del hocico; Ah, altura del hocico; AMP, ancho mínimo postorbital; ARM, altura de la rama mandibular; DAP, diámetro ántero-posterior de los caniniformes y molariformes; DVL, diámetro vestíbulolingual de los caniniformes y molariformes; LMC, longitud maxilocondilar; LMf4-O, longitud del Mf4 al cóndilo occipital; LSD, longitud de la serie dentaria. Húmero. ADH, ancho distal del húmero; APH, ancho proximal del húmero; LMH, longitud máxima. Radio. LMR, longitud máxima del radio. Ulna. LMU, longitud máxima de la ulna. Fémur. ADF, ancho distal del fémur; APF, ancho proximal del fémur; LMF, longitud máxima del fémur. Tibia. LMT, longitud máxima de la tibia. Fíbula. LMf, longitud máxima de la fíbula. Calcáneo. LMC, longitud máxima del calcáneo.

Otras abreviaturas. AP, antes del presente; ca., circa; d, derecho; ICZN, Internacional Commission on Zoologocal Nomenclature, 1999; IH, índice de hipsodoncia; i, izquierdo, ka, miles de años; Ma, millones de años.

\section{PALEONTOLÓGIA SISTEMÁTICA}

\author{
Superorden XENARTHRA Cope, 1889 \\ Suborden PHYLLOPHAGA Owen, 1842 (= Tardigrada \\ Latham \& Davies en Forster, 1795) \\ Familia MYLODONTIDAE Gill, 1872 \\ Subfamilia SCELIDOTHERIINAE Ameghino, 1904
}

Diagnosis. Phyllophaga (= Tardigrada) de tamaño mediano a grande dentro de los Mylodontidae. Cráneo bajo, largo y estrecho; fórmula dentaria 5/4; series de dientes superiores en filas paralelas, de sección triangular, o subelíptico, borde anterior del maxilar se extiende más allá del Cf1; primer diente superior e inferior molarizado y sin diastema entre el primero y segundo diente; Cf1 elíptico en sección transversal; Mf4 es de sección en "T", mf3 trilóbulado. Premaxilar con las ramas laterales más desarrolladas que las ramas mediales. Mano con cuatro o cinco dedos funcionales, falanges ungueales con garras en los dedos I (excepto en Scelidotherium leptocephalum), II y III. Movimiento limitado entre el trapezoides y el Mc I. Húmero con cresta deltopectoral masiva y desarrollada en sentido distal, y foramen entepicondilar (excepto en Catonyx cuvieri). Fémur comprimido en sentido anteroposterior y de contorno cuadrangular, astrágalo con superficie cóncava para el cuboides. Calcáneo con tuber calcis más marcadamente triangular que el de los milodontinos, solo la región más distal del calcáneo contactaría el suelo. Pie pentadáctilo con dígitos rudimentarios, falanges ungueales con garra en los dedos I, II y III; con fusión de la falange proximal y segunda del dedo III.

Comentarios. Este grupo fue propuesto por Florentino Ameghino en 1904, y aunque no designó formalmente el género tipo, de manera indirecta se interpreta que es Scelidotherium, de acuerdo a la raíz del nombre del taxón nominal de nivel de subfamilia (Art. 64 de ICZN). Además, Paula Couto (1953, 1979) menciona que ese es el género tipo, a su vez McKenna \& Bell (1997: 94) indican que el autor de la subfamilia es Ameghino, 1889; sin embargo, cabe mencionar que si bien efectivamente el autor fue Ameghino, la fecha exacta es 1904.

\section{Scelidotherium Owen, 1839}

Especie tipo. Scelidotherium leptocephalum Owen, 1839.

Especies referidas. La especie tipo y Scelidotherium bravardi Lydekker, 1886.

Diagnosis extendida del género. Se diferencia de los otros géneros por poseer cráneo alargado, bajo y estrecho, región de la caja craneana plana, crestas temporales de los parietales paralelas entre sí, no forman cresta sagital marcada, región fronto-nasal estrecha. En vista anterior, el contorno de la abertura nasal es subtriangular con vértice hacia la región del paladar, los maxilares son casi verticales. Proceso lateral del nasal se extiende más allá del borde anterior del maxilar. Longitud de la región predentaria mayor que la de la serie dentaria superior. Con Cf1 y Mf1-Mf4 de prisma simple, subelípticos o levemente subtriangulares, implantados oblicuamente respecto del plano sagital, algo alargados en sentido anteroposterior. Mandíbula grácil, con rama horizontal baja, borde ventral casi recto. Región anterior predentaria de la rama mandibular muy larga, que en vista oclusal es cuadrangular. Quilla mandibular poco evidente. Húmero con diáfisis grácil. Fémur con el tercio distal más ancho que el proximal, la cabeza del fémur no está alineada verticalmente sobre el cóndilo medial. Calcáneo grácil.

Scelidotherium leptocephalum Owen, 1839

(Figuras 1A-E,F; 2E; 3A,C,E,G,I,K,M; Tablas 1-9)

1917. Scelidotherium carlesi Bosca \& Casanoves, p 48-53.

Holotipo. BM(NH) M 16579 (= RCS 3506), porción posterior de cráneo, porciones de hemimandíbulas derecha e izquierda con las series dentarias; BM(NH) M 16584 (= RSC 3519), astrágalo derecho; $\mathrm{BM}(\mathrm{NH}) \mathrm{M} 16583$ (= RSC 3520), astrágalo izquierdo; BM(NH) M 16581 (= RCS 3510), cuatro cuerpos 

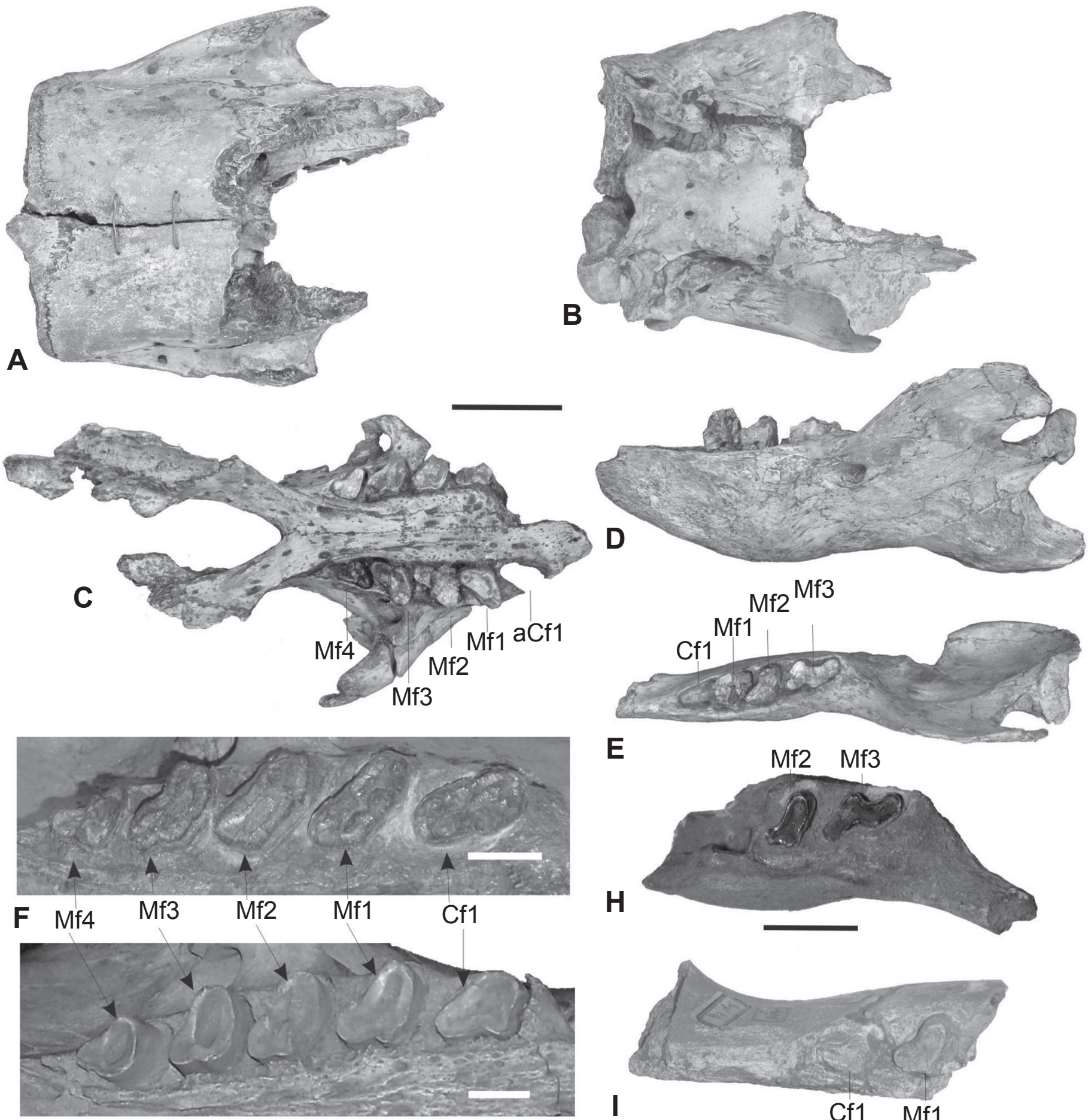

G

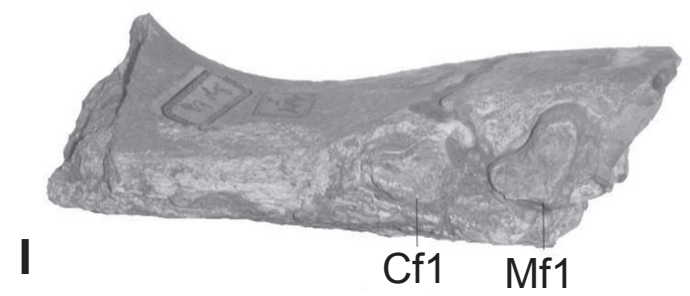

Figura 1. Scelidotherium leptocephalum [BM(NH)M 16579 (= RCS 3506) holotipo]. A, cráneo en vista dorsal; B, en vista ventral; C, palatal en vista oclusal; $\mathbf{D}$ y E, hemimandíbula izquierda en vista externa y vista oclusal. Comparación de la serie dentaria superior derecha. F, Scelidotherium leptocephalum (PIMUZA/V 0508); G, Scelidotherium bravardi (MMP 9-S); Scelidotheriinae indet. (MLP 3-761 holotipo de Scelidotherium floweri Ameghino). $\mathbf{H}$, porción posterior de hemimandíbula izquierda en vista oclusal; I, porción anterior de hemimandíbula izquierda en vista oclusal (MLP 3-440 holotipo de Scelidotherium laevidens Moreno \& Mercerad). Escalas: A-E = 50 mm; F,G = $10 \mathrm{~mm} ; \mathrm{H}, \mathrm{I}=20 \mathrm{~mm}$.

Figura 1. Scelidotherium leptocephalum [BM(NH)M 16579 (= RCS 3506) holotype]. A, skull in dorsal view, B, ventral view, C, palatal in occlusal view, $\mathbf{D}$ and $\mathbf{E}$, left mandible in external view and occlusal view. Comparison of the right upper dental series. F, Scelidotherium leptocephalum (PIMUZ A/V 0508); G, Scelidotherium bravardi (MMP 9-S); Scelidotheriinae indet. (MLP 3-761 holotype Scelidotherium floweri Ameghino). $\mathbf{H}$, posterior portion of left mandible in occlusal view; I, left anterior mandible in occlusal view (Scelidotherium laevidens MLP 3-440 holotype Moreno \& Mercerad). Scale bars: A-E = $50 \mathrm{~mm} ; \mathrm{F}, \mathrm{G}=10 \mathrm{~mm} ; \mathrm{H}, \mathrm{I}=20 \mathrm{~mm}$. 

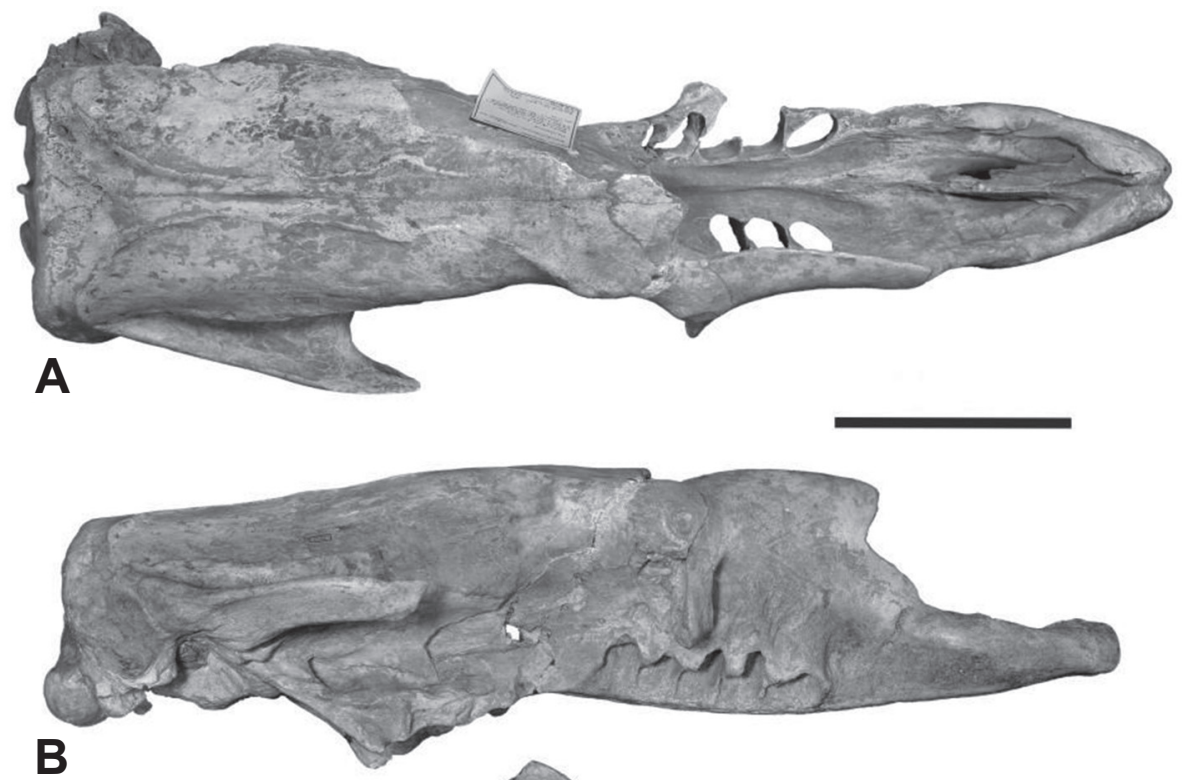

B
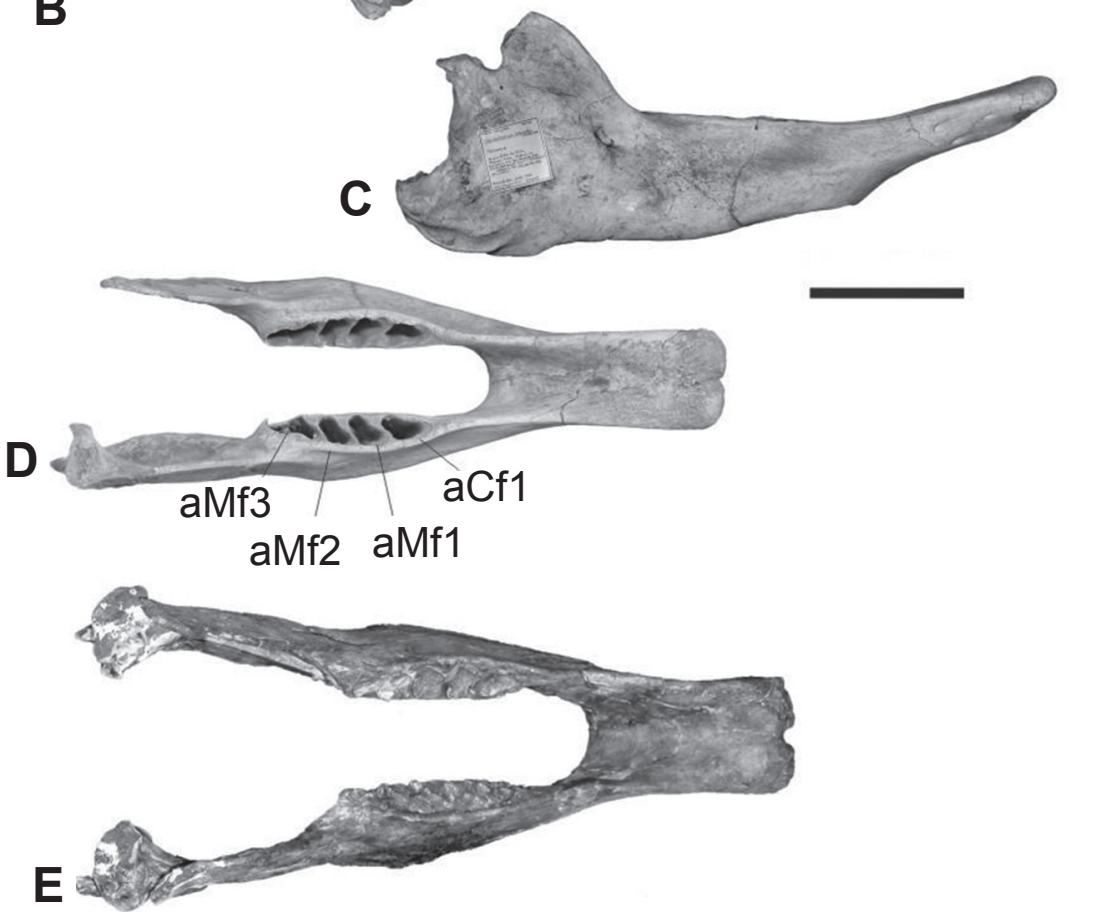

Figura 2. A-D, Scelidotherium bravardi [BM(NH)M 37626 holotipo]: A, cráneo en vista dorsal; B, cráneo en vista lateral derecha; C, mandíbula en vista lateral derecha. D, mandíbula en vista oclusal. E, Scelidotherium leptocephalum (FMNH P 14274) en vista oclusal. Escalas $=100 \mathrm{~mm}$.

Figure 2. A-D, Scelidotherium bravardi [BM(NH)M 37626 holotype]: A, dorsal view skull; B, right side skull; C, jaw right side view; D, jaw occlusal view. E, Scelidotherium leptocephalum (FMNH P 14274) occlusal view. Scale bars $=100 \mathrm{~mm}$.

de vértebras caudales articuladas; $\mathrm{BM}(\mathrm{NH}) \mathrm{M} 16582$ (= RCS 3511), cuatro fragmentos de costillas. Todos estos materiales corresponden a un mismo espécimen.

Localidad tipo y edad. Punta Alta, Bahía Blanca, Buenos Aires, Argentina (Owen, 1839), Pleistoceno tardío.

Diagnosis extendida. Se diferencia de la otra especie por ser el taxón más grande del género. Cf1 elíptico, con un muy leve lóbulo lingual. Dientes superiores más marcadamente elípticos que los inferiores, sin surcos que delimiten lóbulos, emergiendo casi en forma oblicua. Región anterior predentaria de la rama mandibular muy angosta. Fémur con epicóndilo medio más evidente. Mano anterior con dedo I atrofiado y desprovisto de falange ungueal. Calcáneo, en que vista proximal muestra la faceta sustentacular no continua con el cuboides, separadas por hueso no articular. Índice de hipsodoncia es igual a 91 (ver Bargo et al., 2006).

Comentarios. La fecha de publicación de Scelidotherium leptocephalum es Owen, 1839 (Mones, 1985). Mones (1985: 199) realizó correcciones en las fechas de publicación de los trabajos de Owen. De acuerdo a Mones (1985) las contribuciones sobre mamíferos fósiles que realizó Owen están integradas en cuatro fascículos (numerados del 1 al 4) 

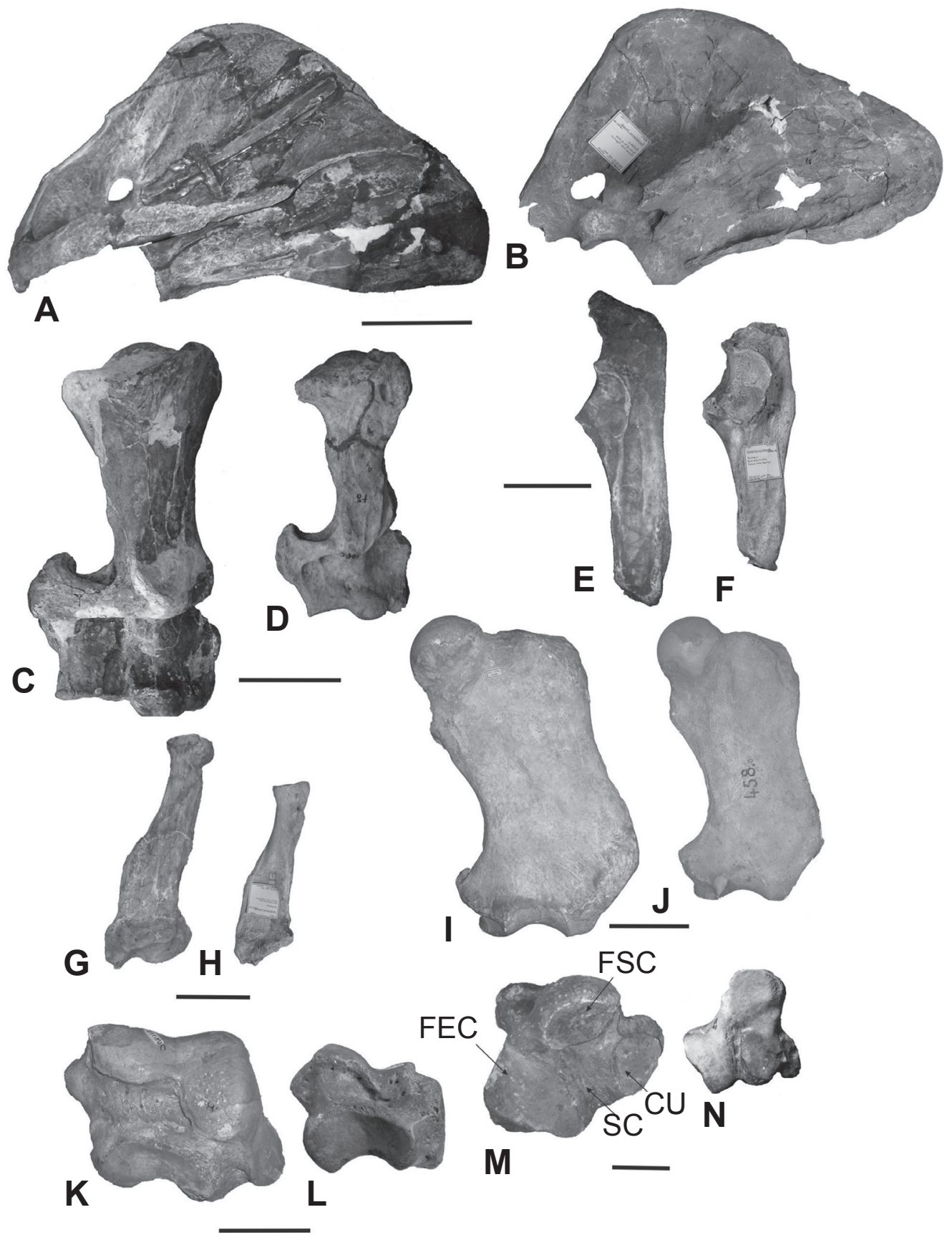

Figura 3. Escápulas, en vista lateral. A, Scelidotherium leptocephalum (MACN 5001) d (a fines comparativos reflejado horizontalmente al lado izquierdo); B, Scelidotherium bravardi [BM(NH)M 37630] d (a fines comparativos reflejado horizontalmente al lado izquierdo); húmeros en vista anterior. C, Scelidotherium leptocephalum (FMNH P 14274) d (a los fines comparativos reflejado horizontalmente al lado izquierdo); D, Scelidotherium bravardi (MLP 3-506) i. Ulnas en vista lateral. E, Scelidotherium leptocephalum (MACN 5001) i; F, Scelidotherium bravardi [BM(NH) M 37631 holotipo] i. Radios. G, Scelidotherium leptocephalum (FMNH P 14274) i (a los fines comparativos reflejado horizontalmente al lado derecho); H, Scelidotherium bravardi (tipo BM(NH)M 37633 d. Fémures en vista anterior. I, Scelidotherium leptocephalum (calco BM(NH) $\mathrm{s} / \mathrm{n}$ ); J, Scelidotherium bravardi (MMP 458-S) i. Astrágalos. K, Scelidotherium leptocephalum (PIMUZ A/V 0509) i; L, Scelidotherium bravardi (MACN 1961) d (a los fines comparativos reflejado horizontalmente al lado izquierdo). Calcáneos derechos en vista proximal. M, Scelidotherium leptocephalum (PIMUZ A/V 0509) en vista medial; N, Scelidotherium bravardi (MACN1921). Escalas: A-J = 100 mm; K-N = 20 mm.

Figure 3. Shoulders, side view. A, Scelidotherium leptocephalum (MACN 5001) d (for comparative purposes reflected horizontally to the left), B, Scelidotherium bravardi (BM(NH) M 37630) d (for comparative purposes reflected horizontally to the left); humerus in anterior view. C, Scelidotherium leptocephalum (FMNH P 14274) d (for comparative purposes reflected horizontally to the left), D, Scelidotherium bravardi (MLP 3-506) i; Ulnas in side view. E, Scelidotherium leptocephalum (MACN 5001) i, F, Scelidotherium bravardi [BM(NH) M 37631 holotype] i; Radios. G, Scelidotherium leptocephalum (FMNH P 14274) i (for comparative purposes reflected horizontally to the right), H, Scelidotherium bravardi holotype [BM(NH) M 3763] d; femora in anterior view. I, Scelidotherium leptocephalum (cast BM(NH) M s/n), J, Scelidotherium bravardi (MMP 458-s) i; Astragalus. K, Scelidotherium leptocephalum (PIMUZ A/V 0509) i, L, Scelidotherium bravardi (MACN 1961) d (for comparative purposes reflected horizontally left). calcaneus rights proximal view. M, Scelidotherium leptocephalum (PIMUZ A/V 0509), N, Scelidotherium bravardi (MACN1921). Scale bars: A-J = $100 \mathrm{~mm}$; K-N = $20 \mathrm{~mm}$. 
Tabla 1. Medidas $(\mathrm{mm})$ del cráneo.

Table 1. Measurements $(\mathrm{mm})$ of the skull.

\begin{tabular}{lccccccc}
\hline Taxón/Especimen & LMC & LSD & AMP & AH & Ah & LMf4-O & AC \\
\hline S. leptocephalum & 520 & 110 & 109 & 100 & 125 & 295 & 117 \\
FMNH P 14274 & 415 & 85 & 95 & 85 & 105 & 230 & 100 \\
MLP 3-409 & 550 & 110 & 100 & 100 & 125 & 290 & 110 \\
MACN 13880 & 515 & 105 & 90 & 90 & 115 & 275 & 100 \\
MACN 13883 & 470 & 105 & 90 & 95 & 105 & 250 & 100 \\
MLP 3-402 & 410 & 90 & 75 & 75 & 113 & 230 & 105 \\
S. bravardi & 500 & 109 & 95 & 95 & 135 & 295 & 112 \\
MMP 9-S & 405 & 100 & 85 & 85 & 116 & 242 & 120 \\
MMP 31-S & 385 & 88 & 80 & 81 & 110 & 213 & 77 \\
MMP 128-S & & & &
\end{tabular}

Tabla 2. Medidas ( $\mathrm{mm})$ de la mandíbula.

Table 2. Measurements $(\mathrm{mm})$ of the jaw.

\begin{tabular}{|c|c|c|c|c|c|c|c|c|c|c|}
\hline Taxón /Espécimen & $\begin{array}{l}\text { DAP } \\
\text { cf1 }\end{array}$ & $\begin{array}{c}\text { DVL } \\
\text { cf1 }\end{array}$ & $\begin{array}{l}\text { DAP } \\
\text { mf1 }\end{array}$ & $\begin{array}{c}\text { DVL } \\
\text { mf1 }\end{array}$ & $\begin{array}{l}\text { DAP } \\
\text { mf2 }\end{array}$ & $\begin{array}{l}\text { DVL } \\
\mathrm{mf} 2\end{array}$ & $\begin{array}{l}\text { DAP } \\
\text { mf3 }\end{array}$ & $\begin{array}{l}\text { DVL } \\
\mathrm{mf3}\end{array}$ & LSD & $\mathrm{ARM}$ \\
\hline $\begin{array}{l}\text { S. bravardi } \\
\text { MMP 705-S }\end{array}$ & 20 & 14 & 14 & 12 & 14 & 12 & 24 & 10 & 83 & 58 \\
\hline MMP 771-S & 16 & 9 & 13 & 14 & 14 & 13 & 23 & 12 & 73 & 56 \\
\hline MMP 157-S & 19 & 15 & 16 & 17 & 17 & 15 & 30 & 12 & 94 & 80 \\
\hline $\begin{array}{l}\text { S. leptocephalum } \\
\text { MMP } 127-\mathrm{S}\end{array}$ & 23 & 13 & 17 & 17 & 18 & 17 & 35 & 14 & 110 & 85 \\
\hline MMP 549 & 23 & 12 & 17 & 17 & 16 & 16 & 33 & 15 & 98 & 80 \\
\hline
\end{tabular}

Tabla 3. Medidas $(\mathrm{mm})$ del húmero. $\left({ }^{*}=\right.$ tomado de Cartelle et al., 2009).

Table 3. Measurements $(\mathrm{mm})$ of the humerus. $\left({ }^{*}=\right.$ taken from Cartelle et al., 2009).

\begin{tabular}{lccc}
\hline Taxón/Espécimen & LMH & APH & ADH \\
\hline $\begin{array}{l}\text { S. leptocephalum } \\
\text { MLP 3-507 }\end{array}$ & 385 & 140 & 200 \\
MMP 614 & 360 & 231 & 160 \\
PIMUZ A/V 0513 & 425 & 160 & 200 \\
PIMUZ A/V 0509 & 350 & 140 & 150 \\
PIMUZ A/V 0508 & 450 & 190 & 225 \\
S. bravardi & 325 & 142 & 162 \\
MLP 3-506 & 400 & 170 & 200 \\
MACN 7154 & 415 & 165 & 180 \\
MACN 14809 & & & \\
\hline
\end{tabular}

Tabla 4. Medidas ( $\mathrm{mm}$ ) del radio.

Table 4. Measurements $(\mathrm{mm})$ of the radius.

\begin{tabular}{lc}
\hline Taxón/Espécimen & LMR \\
\hline S. leptocephalum & $\mathrm{d}, 345$ \\
FMNH P 14274 & $\mathrm{i}, 290$ \\
MLP 3-671 & $\mathrm{d}, 300$ \\
FMNH P 14276 & $\mathrm{d}, 270$ \\
MLP 3-671
\end{tabular}

Tabla 5. Medidas ( $\mathrm{mm}$ ) de la ulna.

Table 5. Measurements $(\mathrm{mm})$ of the ulna.

\begin{tabular}{lc}
\hline Taxón/Espécimen & LMU \\
\hline S. leptocephalum & $\mathrm{d}, 410$ \\
FMNH P 14274 & $\mathrm{i}, 380$ \\
MACN 5001 & $\mathrm{i}, 390$ \\
MACN 7064 & $\mathrm{~d}, 370$ \\
FMNH P 14276 &
\end{tabular}

Tabla 6. Medidas ( $\mathrm{mm}$ ) del fémur. ${ }^{*}=$ tomado de Cartelle et al., 2009). Table 6. Measurements $(\mathrm{mm})$ of the femur. $\left({ }^{*}=\right.$ taken from Cartelle et al., 2009).

\begin{tabular}{lccc}
\hline Taxón/Especimen & LMF & APF & ADF \\
\hline $\begin{array}{l}\text { S. leptocephalum } \\
\text { FMNH P 14276 }\end{array}$ & 450 & 230 & 265 \\
MACN 5001 & 417 & 210 & 222 \\
MACN 7064 & 420 & 210 & 251 \\
MACN 13562 & 380 & 182 & 211 \\
MLP 89-XII-6-4 & 425 & 190 & 230 \\
MLP 3-485 & 385 & 215 & 225 \\
MLP 3-482 & 422 & 225 & 220 \\
MLP 3-484 & 340 & 180 & 170 \\
\hline
\end{tabular}


Tabla 7. Medidas $(\mathrm{mm})$ de la tibia.

Table 7. Measurements $(\mathrm{mm})$ of the tibia.

\begin{tabular}{lc}
\hline Taxón/Espécimen & LMT \\
\hline S. leptocephalum & $\mathrm{d}, 260$ \\
PIMUZ AV 0509 & $\mathrm{i}, 300$ \\
PIMUZ AV 0508 & $\mathrm{d}, 280$ \\
FMNH P 14276 & $\mathrm{~d}, 260$ \\
MACN 5001
\end{tabular}

Tabla 8. Medidas $(\mathrm{mm})$ de la fíbula.

Table 8. Measurements $(\mathrm{mm})$ of the fibula.

\begin{tabular}{lc}
\hline Taxón/Espécimen & LMf \\
\hline S. leptocephalum & $\mathrm{d}, 360$ \\
PIMUZ AV 513 & \\
PIMUZ AV 0509 & $\mathrm{d}, 260$ \\
PIMUZ AV 0508 & $\mathrm{i}, 310$ \\
FMNH P 14276 & $\mathrm{~d}, 290$ \\
MACN 5001 & $\mathrm{d}, 280$ \\
\hline
\end{tabular}

Tabla 9. Medidas ( $\mathrm{mm}$ ) del calcáneo.

Table 9. Measurements $(\mathrm{mm})$ of the calcaneus.

\begin{tabular}{lc}
\hline Taxón/Espécimen & LMC \\
\hline S. leptocephalum & $\mathrm{d}, 200$ \\
MACN 5001 & \\
S. bravardi & $\mathrm{d}, 175$ \\
BM(NH) 37643 & \\
\hline
\end{tabular}

que corresponden a los números: 1, 7, 8 y 13 de la Zoología del Beagle. La cita correcta sobre las descripciones de Scelidotherium es la siguiente: Fascículo VIII (3 de Fossil Mammalia): 65-80, planchas XVIII-XXVII (Mayo de 1839); en lo que se refiere a $S$. leptocephalum las descripciones se encuentran en las páginas: 73-80, planchas XX, XXI, XXIII, XXIV, XXV, XXVI figs. 2-4-6 y XXVII. En el Fascículo XIII (4 de Fossil Mammalia): 81-111, planchas XXVIII-XXXII (Abril de 1840) las descripciones de S. leptocephalum se encuentran en las páginas: 81-99 plancha XXVIII fig. 2.

Materiales referidos a Scelidotherium leptocephalum, procedencia geográfica y estratigráfica. AMNH 11292, porción de hemimandíbula derecha con todos los dientes, Olivera, Buenos Aires, Argentina, Pleistoceno. AMNH 11287, porción de paladar con todos los dientes, Buenos Aires, Pleistoceno. CTES-PZ 7444, hemimandíbula izquierda con dientes, arroyo Toropí, Bella Vista, Corrientes, Argentina, Lujanense. GCF 11, hemimandíbula parcial sin dientes, ciudad de San Pedro, Buenos Aires, Argentina, Lujanense. PVE-F s/n, cráneo y mandíbula parcial, Villa Escolar en las barrancas del río Bermejo, Formosa, Argentina. Formación Río Bermejo, Lujanense. MFCA 757, cráneo, margen derecha del río Paraná, Alvear, Santa Fe, Argentina, Pleistoceno. MLP 3-402, cráneo, Buenos Aires, Pleistoceno, figurado por Lydekker (1895: plancha LV figs. 1 y 1a). FMNH P 14274, cráneo, mandíbula, escápula derecha, humero izquierdo, ulnas derecha e izquierda, radios derecho e izquierdo, algunos huesos de la mano, río Quequén, Buenos Aires, Argentina, Pleistoceno. FMNH P 14267, cráneo, Buenos Aires, Argentina, Pleistoceno. MACN
9625: cráneo parcial, Laguna Vital, Chascomús, Buenos Aires, Argentina, "Pampeano superior" (¿Bonaerense o Lujanense?). MACN 13880, cráneo parcial y MACN 13883, cráneo, ambos especímenes provienen de Necochea, Buenos Aires, Argentina, Pleistoceno. MMP 1155, cráneo y mandíbula, ciudad de Mar del Plata (Av. Constitución y Tejedor, a $3 \mathrm{~m}$ aprox. de profundidad), Buenos Aires, Formación Loberia, Lujanense. MLP s/n, hemimandíbula derecha con toda la serie dentaria, río Quequén Salado, Buenos Aires, Argentina, Lujanense. MLP 3-429, hemimandíbula derecha parcial, río Quequén Salado, Buenos Aires, Argentina, Formación "Pampeano superior" (¿Bonaerense o Lujanense?). MLP 3-671, cráneo, húmero izquierdo, radio izquierdo, mano casi completa izquierda, Olavarría, Buenos Aires, Argentina, Pleistoceno. MLP 68-V-29-1, mandíbula parcial con diente, La Plata (calle 54 entre 4 y 5), Buenos Aires, Argentina, Lujanense. MLP 3-407, cráneo y mandíbula asociado, Pleistoceno. MLP 3-408, cráneo y hemimandíbula derecha, "Pampeano superior". MLP 3-409, cráneo parcial, sin dientes, "Pampeano superior" (¿Bonaerense o Lujanense?). MLP 3-420, cráneo y mandíbula parciales, Formación "Pampeano superior” (¿Bonaerense o Lujanense?). MLP 86-VI-23-94, porción posterior de cráneo, hemimandíbula izquierda sin dientes, Centinela del Mar, Mar del Plata, Buenos Aires, Argentina, nivel eólico 6 (perfil de Goin), Pleistoceno. MLP 52-X-41, maxilar con el palatal, posee Mf1, Mf2, Mf3 y Mf4, hemimandíbula derecha parcial con todos los dientes, río Quequén Salado, Buenos Aires, Argentina, Lujanense. MLP 89-XII-6-4, fémures derecho e izquierdo, La Quesera, Salta, Argentina, Pleistoceno tardío. MPA 85-50-I-D, cráneo parcial con los dientes, Playa del Barco, Pehuen Co, Buenos Aires, Argentina, Bonaerense-Lujanense. PIMUZ A/V 0513, cráneo, mandíbula, vertebras dorsales, clavículas derecha e izquierda, escápula izquierda, ulna derecha, húmero derecho, radio derecho, mano derecha parcial, fémur izquierdo, fíbula derecha y calcáneo izquierdo, San Nicolás, Buenos Aires, Argentina, "Pampeano superior” (¿Bonaerense o Lujanense?). El cráneo y mandíbula fue figurado por Schulthess (1920: lám. 6, fig. 13). PIMUZ A/V 0509, cráneo parcial, mandíbula completa, húmero izquierdo, mano derecha parcial, astrágalo derecho, calcáneo derecho, fémur derecho (parcial) e izquierdo, tibia derecha, fíbula derecha, Arroyo del Medio, Buenos Aires, "Pampeano intermedio", el cráneo y mandíbula fue figurado por Schulthess (1920: lám. 6, fig. 14). PIMUZ A/V 0508, cráneo, húmero izquierdo, ulna derecho, radio derecho, fémur derecho, tibia derecha, fíbula derecha, pie izquierda parcial, pie derecho parcial, astrágalos derecho e izquierdo y calcáneos derecho e izquierdo, Pergamino, Buenos Aires, Argentina, "Pampeano intermedio".

Materiales referidos a cf. Scelidotherium leptocephalum. MACN 7064, fémures derecho (completo) e izquierdo (solo la diáfisis), ulnas derecha e izquierda, Chascomús, Buenos Aires, Pleistoceno. MLP 3-507, húmero izquierdo seccionado longitudinalmente, Formación "Pampeano superior". PIMUZ A/V 0506, hemimandíbula izquierda sin dientes, escápula izquierda, tibia derecha, una vértebra dorsal, de un individuo juvenil, arroyo Pergamino, Buenos Aires, Argentina, 
Pleistoceno. PIMUZ A/V 0526, porción anterior de cráneo, astrágalo izquierdo, barranca del río Paraná, San Lorenzo, Buenos Aires, Argentina, "Pampeano intermedio". PIMUZ A/V 4149, porción posterior de cráneo, Baradero, Buenos Aires, Argentina, "Pampeano superior” (¿Bonaerense o Lujanense?). Descripción del holotipo. El fragmento anterior del cráneo tipo, preservó parcialmente el pterigoideo derecho, palatinos derecho e izquierdo, paladar con el alvéolo del Cf1 izquierdo, y los Mf1 a Mf4 derechos e izquierdos. En vista palatal, se observa que la sutura entre el maxilar y el palatino es rectilínea a nivel de Mf2 y Mf3, además de la sutura de los maxilares que es paralela a las series de dientes. La base del proceso cigomático del maxilar se encuentra a nivel de los Mf2 y Mf3. En sección los molariformes Mf1, Mf2 y Mf3 son subelípticos, con leves surcos linguales longitudinales. El Mf4 es el más pequeño de la serie, en sección es el más subtriangular.

El fragmento posterior del cráneo tipo de Scelidotherium leptocephalum $\mathrm{BM}(\mathrm{NH}) \mathrm{M} 16579$ (= RCS 3506) en vista dorsal no muestra la cresta parasagital, es evidente la sutura parieto-supraoccipital y la sutura fronto-escamosal; esta última sutura se encuentra por detrás del extremo más anterior de la apófisis cigomática del escamoso. Se preservan ambos escamosos, aunque solo el izquierdo conserva la apófisis cigomática. La cresta occipital posee escaso desarrollo.

En vista ventral, se observa la sutura rectilínea entre el basioccipital y basiesfenoides, con escaso desarrollo del proceso paraoccipital y de la fosa glenoidea izquierda. El foramen cóndilar del occipital es poco visible, el entotimpánico tiene el aspecto de una lámina muy delgada. El proceso cigomático del maxilar se encuentra entre los Mf2-Mf3.

En vista lateral izquierda, se observa que la sutura entre el escamoso y el parietal es horizontal.

En vista posterior, solo se preservó el cóndilo occipital derecho. El occipital es de contorno semicircular, la región supraoccipital es levemente plana, la protuberancia occipital externa presenta escaso desarrollo.

La mandíbula tipo BM(NH) M 16579 no preservó la zona de la sínfisis, ambas hemimandíbulas están separadas. La hemimandíbula izquierda no conservó el cfl, en tanto el mfl, $\mathrm{mf} 2$ y $\mathrm{mf} 3$ se preservaron parcialmente; la hemimandíbula derecha se encuentra seccionada en sentido longitudinal. En sección, el alvéolo del cfl presenta un suave lóbulo en posición labial que indicaría que estuvo presente un lóbulo en el prisma del cfl; el mf2 y mf3, presentan un leve surco vestibular longitudinal; el $\mathrm{m} 3$ presenta un surco lingual profundo y otro vestibular, ambos longitudinales.

Además, se preservaron cuatro cuerpos vertebrales de vértebras caudales, y fragmentos de costillas.

Ambos astrágalos $[\mathrm{BM}(\mathrm{NH}) \mathrm{M} 16584$ (= RSC 3519) derecho y $\mathrm{BM}(\mathrm{NH}) \mathrm{M} 16583$ (= RSC 3520] izquierdo, muestran en vista fíbular, una leve concavidad en la faceta fíbular. En vista dorsomedial, muestran un proceso central odontoide poco prominente. La faceta discoidea está poco desarrollada. El ángulo entre la faceta discoidal y la faceta odontoidea es de $160^{\circ}$ aproximadamente.
Tipo de los sinónimos de Scelidotherium leptocephalum, procedencia y comentarios

Scelidotherium carlesi Bosca y Casanoves, 1917

Holotipo. MCNV 64-7, esqueleto casi completo.

Localidad tipo y edad. Río Salado del Sur, Buenos Aires, Argentina, Pleistoceno.

Comentarios. De acuerdo a Bosca y Casanoves (1917) el tipo consta de cráneo, mandíbula, varias vertebras cervicales, dorsales, caudales y otros huesos del poscráneo, en buen estado de preservación. Sin embargo, Bosca y Casanoves (1917) no menciona caracteres diagnósticos que justifiquen esta nueva especie. Luego de revisar el ejemplar, y ya que tampoco hemos identificado caracteres morfológicos que justifiquen un nuevo taxón, coincidimos con la propuesta de McDonald (1987) sobre la sinonimia de este taxón con $S$. leptocephalum.

En cuanto a la procedencia geográfica y estratigráfica del material tipo de $S$. carlesi, Bosca y Cacanoves (1917) provee muy escasa información. Sin embargo, de acuerdo con el esquema estratigráfico hoy vigente, los yacimientos fosilíferos del río Salado son portadores de mamíferos fósiles que corresponden al Bonaerense ("Pampeano superior" de Ameghino, 1881) y al Lujanense ("Pampeano lacustre" de Ameghino, 1881) (Krmpotic y Scillato- Yané, 2007). Por lo tanto la edad de los sedimentos portadores del material tipo estaría comprendida entre el Pleistoceno medio al Pleistoceno tardío.

Scelidotherium bravardi Lydekker, 1886

(Figuras 1G; 2A-D; 3B,D,F,H,J,L,N; Tablas 1-3, 9,10)

1908. Scelidotherium chapalmalense Ameghino, p 426.

Holotipo. BM(NH) M 37626, cráneo; BM(NH) M 37649, mandíbula sin los dientes; $\mathrm{BM}(\mathrm{NH}) \mathrm{M} 37630$, escápula derecha; $\mathrm{BM}(\mathrm{NH}) \mathrm{M}$ 37631, ulna izquierda; $\mathrm{BM}(\mathrm{NH})$ M 37632, radio derecho; $\mathrm{BM}(\mathrm{NH}) \mathrm{M}$ 37634, escafoides izquierdo; $\mathrm{BM}(\mathrm{NH}) \mathrm{M} 37635$, ectocuneiforme; $\mathrm{BM}(\mathrm{NH}) \mathrm{M}$ 37636, Mc I izquierdo; BM(NH) M 37643, calcáneo derecho; $\mathrm{BM}(\mathrm{NH}) \mathrm{M} 37644$, calcáneo izquierdo; $\mathrm{BM}(\mathrm{NH}) \mathrm{M} 37645$, Mc III; BM(NH) M 37646-47, sesamoides. De acuerdo a la ficha que acompaña al material tipo ellos fueron colectados por Bravard en 1854.

Localidad tipo y edad. "Toscas" del Río de La Plata, Buenos Aires, Argentina, Formación Ensenada.

Diagnosis extendida. Morfología similar a Scelidotherium leptocephalum, pero de tamaño menor. Cráneo más estrecho que $S$. leptocephalum, dientes superiores triangulares en sección. Mandíbula con región predentaria más estrecha y más dorsal que S. leptocephalum, $\mathrm{mf} 3$ con lóbulo posterior recto y no como en el homólogo de $S$. leptocephalum que es más curvado en sección en forma de "C". El postcráneo más grácil que $S$. leptocephalum. Húmero con foramen entepicondílio doble. Calcáneo, en vista proximal con faceta sustentacular levemente continua con el cuboides. Índice de hipsodoncia es igual a 75 (Tabla 10).

Comentarios. El espécimen tipo es el más completo Scelidotheriinae del Piso/Edad Ensenadense de Argentina 


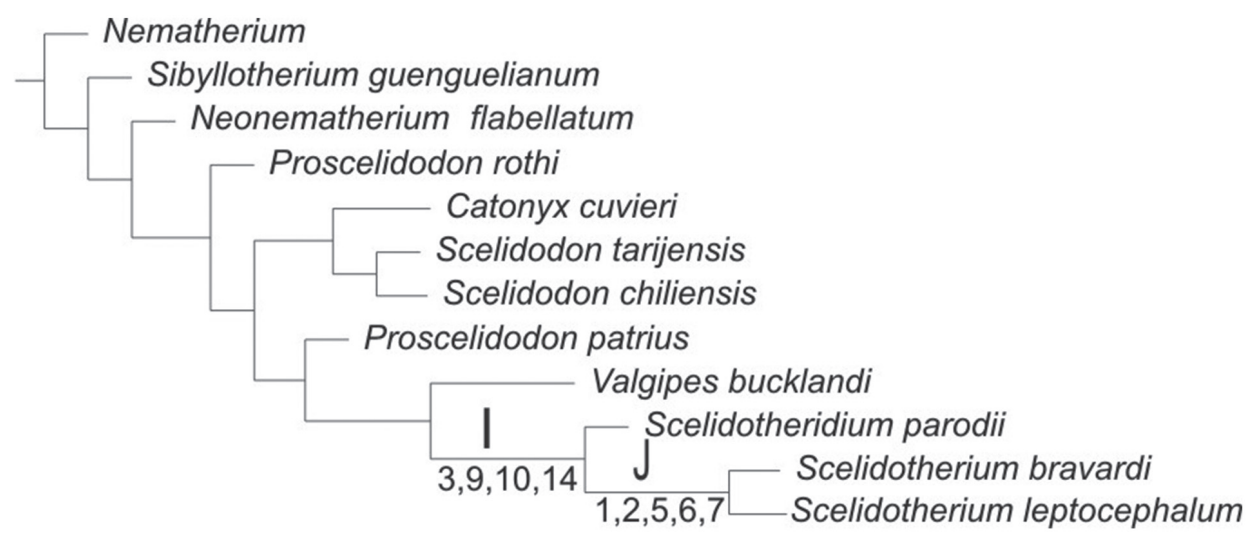

Figura 4. Árbol de máxima parsimonia $(L=34 ; I C=0,765 ; I R=0,8 ; I C R=0,612)$ mostrando la relaciones filogenéticas de Scelidotherium con los restantes Scelidotheriinae. Las letras indican los nodos y los números las sinapomorfias (ver Miño-Boilini, 2012a: 182).

Figura 4. Maximum parsimony tree $(\mathrm{L}=34, \mathrm{Cl}=0.765, \mathrm{RI}=0.8, \mathrm{ICR}=0.612)$ showing the phylogenetic relationships of the remaining Scelidotherium Scelidotheriinae. The letters indicate the numbers of nodes and the synapomorphies (see Miño-Boilini, 2012a: 182).

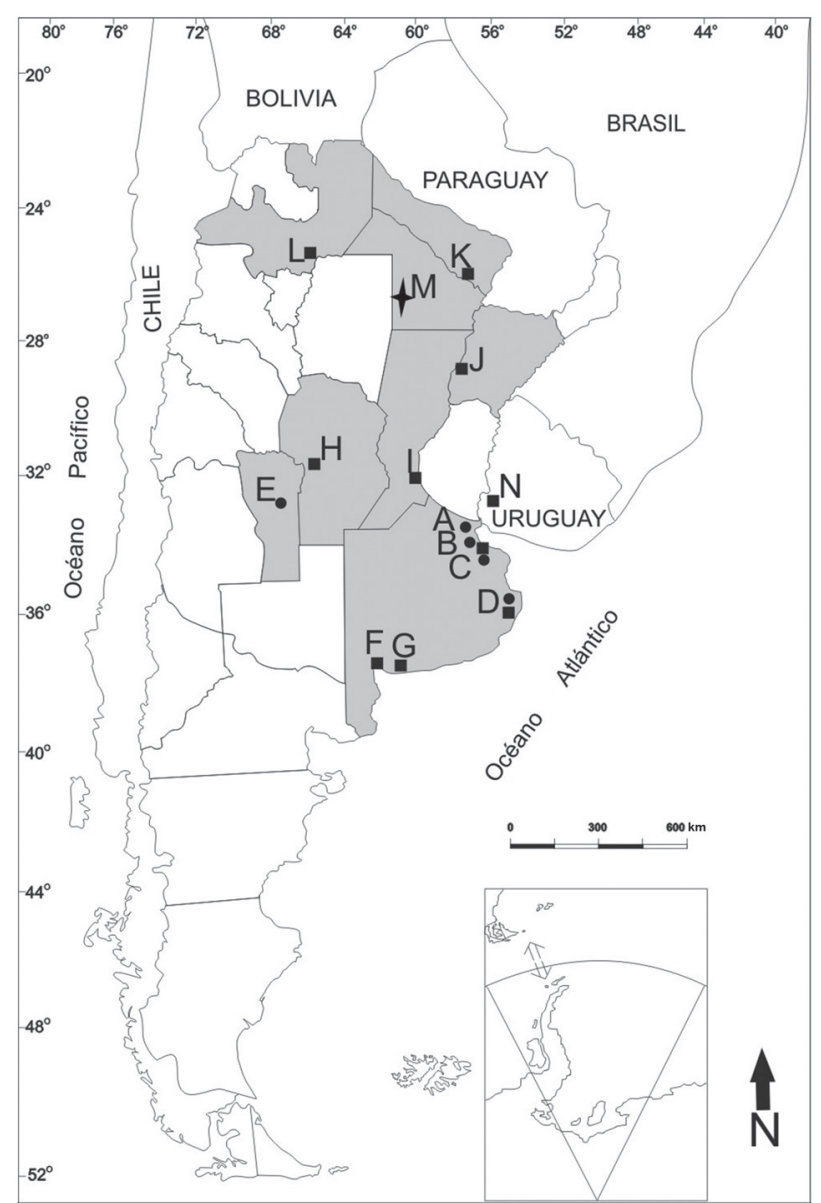

Figura 5. Distribución geográfica de Scelidotherium en Argentina y Uruguay. Estrella, Scelidotherium sp.; círculo, S. bravardi; rectángulo, S. leptocephalum. A, "Toscas" del Río de La Plata; B, Olivos; C, Mar del Plata; D, La Plata; E, Villa Larca; F, Punta Alta; G, río Quequén Salado; H, Elena; I, Alvear; J, Bella Vista; K, Rosario de la Frontera; L, Villa Escolar; M, Charata; N, Soriano.

Figura 5. Geographic distribution of Scelidotherium in Argentina and Uruguay. Star, Scelidotherium sp.; circle, S. bravardi; rectangle, S. leptocephalum.
Tabla 10. Índice de hipsodoncia de Scelidotherium bravardi. Tabla 10. Hypsodonty index of the Scelidotherium bravardi.

\begin{tabular}{lccc}
\hline S. bravardi & LSR & ARM & IH \\
\hline MMP 458-S & 82 & 60 & 73 \\
MMP 157-S & 94 & 80 & 85 \\
MACN 2289 & 75 & 56 & 74 \\
MMP 705-S & 83 & 58 & 69 \\
MMP 771-S & 73 & 56 & 76 \\
& n: 5 & & Media $=75$ \\
\hline
\end{tabular}

conocido. Lydekker (1886) erigió esta especie, luego en 1895 mencionó que podría ser es un sinónimo de Scelidotherium leptocephalum. Este autor sugirió que probablemente las diferencias del cráneo tipo [BM(NH) M 37626] con los especímenes de $S$. leptocephalum se corresponden en realidad un posible dimorfismo sexual.

Teniendo en cuenta que el material tipo se encuentra en buen estado de preservación, además consta de varias partes del esqueleto, esta especie debe ser considerada válida, ya que presenta caracteres diferenciales a nivel del cráneo, mandíbula y post-cráneo (ver más arriba).

En cuanto a la procedencia geográfica y edad, en la etiqueta que acompaña al tipo dice: "Banks of the Rio Plata", lo que indica que procede de las "Toscas" del Río de La Plata, Buenos Aires. Según Soibelzon et al. (2008) la Formación Ensenada correspondiente a las "Toscas" del Río de La Plata" se depositaron probablemente durante el evento Jaramillo (cron C1r 1n, ca. de $1 \mathrm{Ma}$, Pleistoceno temprano-medio).

Materiales referidos a Scelidotherium bravardi, procedencia geográfica y estratigráfica. MMP 9-S, cráneo parcial, al NE de la ciudad de Mar del Plata frente a la Playa Santa Elena, Partido de General Pueyrredon, Buenos Aires, Argentina, Ensenadense. MMP 31-S, cráneo y mandíbula parcial, Barranca costera frente al Parque Camet, Mar del Plata, Buenos Aires, Argentina, Ensenadense, la ficha de ingreso menciona 
que procede de Formación Vorohué (base del acantilado), actualmente y de acuerdo a nuevos estudios (ver Bidegain et al., 2005; Cione et al., 2007:263) las sedimentitas más antiguas del Norte de la ciudad de Mar del Plata corresponden al Piso/Edad Ensenadense, por lo tanto y de acuerdo a que proviene de los niveles basales del acantilado se interpreta que corresponde a la Formación Miramar. MMP 128-S, cráneo sin dientes, Mar del Plata, Buenos Aires, Argentina, de la base de la barranca frente al tambo del campo de Cobo, Formación Miramar. MMP 458-S, cráneo, mandíbula, fémur izquierdo, radio derecho, rotula, barranca costera, $800 \mathrm{~m}$ al NNE del caño de desagüe, Mar del Plata, Buenos Aires, Argentina, Ensenadense. MMP 157-S, cráneo y mandíbula, Playa Estrada, Mar del Plata, Buenos Aires, Argentina, Ensenadense. MACN 2289, hemimandíbula derecha parcial con toda la serie dentaria, "Toscas" del Río de La Plata, Olivos, ciudad de Buenos Aires, Argentina, Formación Ensenada. MACN 2298, astrágalo derecho, "Toscas" del Río de La Plata, Olivos, ciudad de Buenos Aires, Argentina, Formación Ensenada. MACN 1961, astrágalos derecho e izquierdo, "Toscas" del Río de La Plata, Olivos, ciudad de Buenos Aires, Argentina, Formación Ensenada. MACN 1921, calcáneo parcial, "Toscas" del Río de La Plata, Olivos, ciudad de Buenos Aires, Argentina, Formación Ensenada. MMP 705, hemimandíbula derecha parcial solo preservo el cf1, $\mathrm{mf} 2$ y $\mathrm{mf} 3$, frente a la escuela de artillería, Mar del Plata, Buenos Aires, Argentina, Formación Miramar. MMP 771-S, hemimandíbula derecha parcial con toda la serie dentaria, frente a la Playa Santa Elena, ciudad de Mar del Plata, Buenos Aires, Argentina, Ensenadense. MLP 3-411, cráneo y mandíbula parciales, Ensenadense.

Materiales referidos a cf. Scelidotherium bravardi. MMP 17-S, hemimandíbula izquierda parcial, barrancas costeras entre Mar del Plata y Mar Chiquita (Asilo Unzué), Mar del Plata, Buenos Aires, Ensenadense. MLP 3-506, húmeros derecho e izquierdo (presentan foramen entepicondilio doble), Ensenadense, en todos los húmeros de S. leptocephalum no se observa esta característica, solo en este espécimen, por lo que podría tratarse de un carácter diagnostico de S. bravardi, dado que el material proviene del "Pampeano inferior". MACN 7150, húmero izquierdo, ulna parcial, fémur derecho parcial, ciudad de Buenos Aires (excavaciones del Puerto Nuevo), Buenos Aires, Argentina, Ensenadense. MLP 52-X4-9, porción distal de húmero izquierdo, Punta Hermengo, Miramar, Buenos Aires, Argentina, Ensenadense. MLP 3-412, cráneo parcial en mal estado de preservación, Ensenadense. MLP 96-VIII-1-2, hemimandíbula izquierda parcial sin dientes, cantera Hernández (calle 516 entre 30 y 31), ciudad de La Plata, Buenos Aires, Argentina, Ensenandense. MLP $\mathrm{s} / \mathrm{n}$, hemimandíbula derecha con toda la serie dentaria, cantera Hernández (calle 514), ciudad de La Plata, Buenos Aires, Argentina, a $10 \mathrm{~m}$ de profundidad y a $2,5 \mathrm{~m}$ por debajo de la calcita. MMP 3986, fémur derecho parcial, tibia y fíbula derechas, cava 386, Mar del Plata, Buenos Aires, Ensenadense, este material procede los niveles donde fueron hallados Artotherium aungustidens Gervais \& Ameghino, 1880 y Glyptodon munizi Ameghino, 1881 ambos taxones restringidos al Piso/Edad Ensenadense (Soibelzon et al., 2006). MACN
1960, hemimandíbula derecha parcial, solo preservo mf1, mf2 y mf3, "Toscas" del Río de La Plata, Olivos, ciudad de Buenos Aires, Argentina, Ensenadense. MACN 1891, hemimandíbula izquierda parcial con cfl y mf1, "Toscas" del Río de La Plata, Olivos, ciudad de Buenos Aires, Argentina, Ensenadense.

Descripción del holotipo. El cráneo tipo de Scelidotherium bravardi [BM(NH) M 37626], no preservó los dientes, solo sus alvéolos, ni el escamoso izquierdo y los nasales, en tanto que el maxilar izquierdo solo es parcial. En vista dorsal, se observan las crestas parasagitales no forman una cresta sagital como en S. leptocephalum son paralelas entre sí y finalizan a nivel de los procesos postorbitales. Las suturas craneanas aún son visibles, la más evidente es la parieto-supraoccipital, lo que indicaría que el animal fue un adulto joven. La sutura fronto-parietal se encuentra por detrás de la apófisis cigomática del inicio del escamoso como en S. leptocephalum. La sutura del naso-frontal es en cuña con el vértice hacia el frontal, como en S. leptocephalum. Se observa una leve constricción transversal a nivel de los frontales, en S. leptocephalum dicha constricción es menor. En vista lateral derecha, se aprecia el hundimiento de los parietales, que forma la fosa temporal sin embargo, en Scelidotherium leptocephalum el hundimiento es menos evidente. La sutura que separa el escamoso del parietal es horizontal. El pterigoideo se encuentra roto en su porción más ventral, describe un contorno subtriangular, y se articula con el aliesfenoides. El lagrimal está bien desarrollado y es de contorno subcircular; el foramen lagrimal es subcircular como en S. leptocephalum. La porción descendente de la región cigomática del maxilar es casi vertical sin embargo, en S. leptocephalum es más oblicua. El paladar es casi plano en S. leptocephalum es más convexo, el premaxilar está muy desarrollado como en $S$. leptocephalum, a su vez no se observan las suturas con el maxilar. En vista posterior, el plano occipital es de contorno semicircular, el foramen magnum es póstero-ventral como en Scelidotherium leptocephalum, la cresta occipital y la protuberancia externa media del occipital poseen escaso desarrollado como en S. leptocephalum. Los dientes superiores son de sección más triangulares que los de S. leptocephalum. En vista dorsal, en la mandíbula del tipo $[\mathrm{BM}(\mathrm{NH}) \mathrm{M} 37649)]$ se aprecia que la sínfisis es muy estrecha y más rectangular y no tan cuadrangular como en $S$. leptocephalum, y posee una longitud mayor que la de la serie dentaria. El proceso cóndilar es de contorno subtriangular como en S. leptocephalum. En vista lateral, el cóndilo mandibular se encuentra por encima de la superficie oclusal de la serie dentaria sin embargo, en S. leptocephalum se encuentra levemente al mismo nivel del plano oclusal. El borde ventral de la rama horizontal es casi recto, con una leve curvatura a nivel del mf2-mf3, sin embargo, en S. leptocephalum dicha curvatura es levemente más convexa. La porción anterior de la rama horizontal es levemente más dorsal que la de $S$. leptocephalum. El proceso angular del dentario presenta definidos surcos en la superficie externa como en $S$. leptocephalum, seguramente para la inserción del músculo masetero. En vista lateral, la escápula es similar a la de $S$. leptocephalum, es de contorno aproximadamente triangular, la espina se encuentra rota a nivel del puente. La fosa supraespinosa es levemente más amplia que la infraespinosa y subtriangular, a su vez la fosa 
infraespinosa es de contorno subrectangular. El radio del tipo [BM(NH) M 37633] en vista medial presenta un gran desarrollo de la tuberosidad para inserción del biceps brachii como en S. leptocephalum. El borde interno presenta una leve convexidad en S. leptocephalum dicho borde es más convexo. En el extremo distal, se observa el escaso desarrollo de la apófisis estiloides. El calcáneo [BM(NH) M 37643] es más grácil que el de $S$. leptocephalum a su vez en vista tibial se observa que la faceta sustentacular es continua con la del cuboides.

\section{Tipo de los sinónimos de Scelidotherium bravardi, procedencia y comentarios}

Scelidoherium chapalmalense Ameghino, 1908

Holotipo. Hemimandíbula derecha con toda la serie dentaria. Localidad tipo. Buenos Aires, Argentina.

Comentarios. Esta especie no fue tratada en la última revisión realizada por McDonald (1987). Kraglievich (1923) mencionó lo siguiente sobre la procedencia del espécimen tipo: "es que el aspecto de la fosilización de la pieza y ciertas adherencias de tosca, parecen indicar que este resto fósil no proviene del horizonte chapadmalalense y sí, con más probabilidad, del horizonte pampeano inferior o ensenadense....por lo que he podido averiguar que el ejemplar fue exhumado por empleados del Museo de La Plata, cerca de la localidad de Miramar, donde a causa afloran los horizontes, chapadmalalense y ensenadense".

De acuerdo a Mones (1986) el holotipo debería estar depositado en las colecciones del MACN, sin embargo está perdido. Rovereto (1914, lám. XXVI, fig. 8) figuró por vez primera el ejemplar tipo; este autor no realizó ningún comentario sobre la especie, solo la mencionó como fósil del Chapadmalalense (Rovereto, 1914, p. 205). Kraglievich (1923) entre los comentarios que hizo sobre este taxón, concluyó que corresponde a Scelidotherium, pero de antigüedad Ensenadense y no Chapadmalalense, como lo había asumido Ameghino en 1908. Luego, Kraglievich (1945) reestudió dicho especimen tipo y figuró un nuevo material de S. chapalmalalense, que corresponde a una hemimandíbula izquierda, procedente de Miramar (colección de KraglievichRoth, 4 figs. 1-3). Además, figuró el tipo de $S$. chapalmalense (Kraglievich, 1945, fig. 4), que de acuerdo a él el tipo está depositado en MLP bajo el número MLP 3-848, y no como sostiene Mones (1986) en el MACN. Kraglievich (1945) concluyó que el tipo procede del Ensenadense, y con dudas menciona que podría corresponder a un juvenil de $S$. bravardi.

Cabe mencionar que tanto la mandíbula que figura Rovereto (1914, lámina XXVI fig. 8), como aquella de Kraglievich (1945, fig. 4), corresponden a una hemimandíbula derecha con toda la serie dentaria; son similares en la morfología dentaria, pero la que figuró Rovereto está fragmentada a nivel de la porción ascendente, y la figurada por Kraglievich (1945, fig. 4) presenta dicha porción de la rama ascendente; esto deja la duda sobre si en realidad corresponden al mismo espécimen.

En resumen, todo indica que Scelidotherium chapalmalense procede de la Formación Miramar asignada al Piso/Edad Ensenadense. A partir de la presente revisión, la especie es considerada un nomen dubium teniendo en cuenta que no posee caracteres diagnósticos claros que justifiquen un nuevo taxón. A su vez S. chapalmalense es un sinónimo de S. bravardi, coincidiendo con lo propuesto por Kraglievich (1945), ya que en el mf3 el lóbulo posterior es recto y no curvado como en $S$. leptocephalum.

\section{Otros escelidoterinos referidos a Scelidotherium}

Los taxones mencionados en la siguiente lista fueron nominados e incluidos en el género Scelidotherium, aunque cabe mencionar estas especies deben ser consideradas Scelidotheriinae indet. por distintos motivos.

Scelidotherium floweri Ameghino, 1881

Holotipo. MLP 3-761, porción posterior de hemimandíbula izquierda sólo preserva el mf2 y mf3 (Figura 1H).

Localidad tipo. Luján, Buenos Aires, Argentina.

Comentarios. Ameghino (1889, p. 724) mencionó que fundó esta especie sobre ..."una rama horizontal de la mandibula inferior y un maxilar superior, ambas piezas con casi toda la dentadura, provenientes de un individuo adulto...". Lamentablemente solo fue hallada la hemimandíbula como parte del material tipo y carece de cualquier morfología que permita una asignación específica concreta. Hoffstetter (1952) mencionó que esta es una especie mal conocida, a su vez Lydekker (1895) y McDonald (1987) sugirieron que es un sinónimo de Scelidotherium leptocephalum, sin embargo, no mencionan la justificación de dicha sinonimia.

En cuanto a la edad de los sedimentos portadores del material y según Ameghino (1889) procede del "Piso Belgranense" de la "formación pampeana".

Según la presente revisión esta especie es declarada como nomen dubium (ICZN, 1999).

\section{Scelidotherium bellulum Ameghino, 1886}

Holotipo. Según Ameghino (1889) el tipo corresponde al último diente de la serie dentaria superior.

Localidad tipo. Barrancas de los alrededores de la ciudad de Paraná, Entre Ríos, Argentina.

Comentarios. Ameghino (1889) figuró y describió el espécimen (plancha XI figs. 13, 13a). En el MACN se encuentra un molde del material tipo (MACN A 1583). De acuerdo a McDonald (1987, p. 184) este taxón es un sinónimo junior de Scelidotherium leptocephalum. En sección es ligeramente más subcilíndrico que el homólogo de $S$. leptocephalum y presenta un leve surco longitudinal.

A su vez, el mismo Ameghino (1886) dudó sobre la incorporación de esta especie en el género Scelidotherium, esto se ve en la grafía de su asignación: "?Scelidotherium bellulum" Ameghino (1886). En esta revisión consideramos la especie como nomen dubium (ICZN, 1999), ya que el material es pobre y sus caracteres no son suficientes para una diagnosis.

Teniendo en cuenta lo fragmentario del material tipo no coincidimos con la hipótesis propuesta por McDonald (1987) sobre la probable sinonimia de este taxón con Scelidotherium leptocephalum.

Scelidotherium laevidens Moreno \& Mercerat, 1891 
Holotipo. MLP 3-440, hemimandíbula izquierda preserva el cf1-mf1 (fig. 1I).

Localidad tipo. Catamarca, Argentina.

Comentarios. Desde que Moreno \& Mercerat (1891) fundaron esta especie no se han referido materiales a dicho taxón. El material tipo es muy fragmentario y poco diagnóstico. no esta en Ameghino (1898) incluyó esta especie en Scelidodon.

referencias De acuerdo a la revisión efectuada se la considera como nomen dubium (ICZN, 1999). Hasta el momento no existen nuevos registros de escelidoterinos cuaternarios en la provincia de Catamarca (Miño-Boilini \& Carlini, 2009).

\section{DISCUSIÓN}

\section{Sistemática y taxonomía}

Los escelidoterinos cuaternarios están representados por cuatro géneros: Scelidotherium, Scelidodon, Valgipes, y Catonyx (Miño-Boilini, 2012a,b).

Según McDonald \& Perea (2002) el género Scelidotherium está representado por dos especies, S. parodii y S. leptocephalum. Sin embargo, la situación de $S$. parodii es la siguiente; fue erigida por Kraglievich en 1923, sobre un cráneo parcialmente preservado (MACN 5108); tiempo después este mismo autor utilizó a este ejemplar como especie tipo para crear Scelidotheridium parodii Kraglievich, 1934 (ver Pascual et al., 1966; Aramayo, 1988). Sin embargo, McDonald (1987), y McDonald \& Perea (2002), no aceptaron la postura de Kraglievich (1934) e incluyeron a esta especie en Scelidotherium, según la propuesta inicial de Kraglievich (1923). A partir de la revisión realizada, debe considerarse a Scelidotheridium parodii (Pascual et al., 1966; Scillato-Yané et al., 1995), ya que Scelidotheridium se diferencia de Scelidotherium por presentar una talla menor, rostro más corto, en vista anterior, los nasales, maxilares y paladar poseen un contorno circular, el premaxilar no está hipertrofiado, los dientes superiores con surcos más marcados, de sección más triangular (Miño-Boilini, 2012a).

Según el análisis filogenético realizado por MiñoBoilini (2012a) (Figura 4), Scelidotherium (con dos especies válidas, $S$. leptocephalum y $S$. bravardi) es un grupo natural (Nodo J) que se encuentra más relacionado con Scelidotheridium parodii, esta especie es el grupo hermano de Scelidotherium. Este clado está integrado por Scelidotheridium y Scelidotherium sustentado por cuatro sinapomorfias (Nodo I): surco palatino ausente, ausencia de cresta media del basiesfenoides, ausencia del canal entre el proceso paraocipital y mastoideo, e inclinación de la porción anterior de la rama mandibular entre $0^{\circ}$ a $17^{\circ}$.

Desde un punto de vista sistemático, en este trabajo varios taxones son considerados no válidos o dudosos, por lo tanto se redujo significativamente el número de taxones nomimados a solo dos, Scelidotherium leptocephalum y S. bravardi.

\section{Bioestratigrafia y paleobiogeografia}

El género Scelidotherium se registra solamente en sedimentitas del Pleistoceno-Holoceno temprano del actual territorio de la Argentina y Uruguay (Miño-Boilini \& Carlini, 2009; Corona \& Perea, 2010a,b) (Figura 5).
En la Argentina (Figura 5), Scelidotherium se registra en el territorio de las provincias de Buenos Aires, Chaco, Córdoba, Corrientes, Entre Ríos, Salta, San Luis, Santa Fe y Formosa. Sin embargo, los mejores registros en cantidad y calidad de especímenes proceden la región Pampeana (más precisamente Buenos Aires y Córdoba), por lo que interpretamos que estuvo seguramente adaptado a ambientes áridos o semi-áridos y fríos a templado-fríos (Cione \& Tonni, 1995). Los registros fuera de esta región son muy escasos en áreas que durante la mayor parte del Cuaternario (e.g. Mesopotamia argentina, sector occidental de Uruguay y sur de Brasil) poseyeron climas más húmedos y cálidos (Carlini et al., 2004, 2008; Ferrero \& Noriega, 2009; Tonni, 2009), por lo que el género tendría una mayor tolerancia ambiental o la capacidad de dispersarse rápidamente en cortos períodos benignos para sus individuos.

Según esta revisión, hasta el momento Scelidotherium bravardi se encuentra registrada a las sedimentitas del Piso/Edad Ensenadense (ca. 1 Ma-0,4 Ma AP; Pleistoceno temprano-medio) de la provincia de Buenos Aires, más precisamente de las “Toscas” del Río de La Plata (Soibelzon et al., 2010). También fue hallada en sedimentos ensenadenses de la ciudad de Mar del Plata, y ciudad de La Plata, y en el Pleistoceno s.l. de la provincia de San Luis (Miño-Boilini \& Carlini, 2009, Miño-Boilini, 2012a). Cabe mencionar que la especie fue mencionada por Rusconi (1936) para el Ensenadense, sin embargo, ese dato no fue considerado en contribuciones posteriores (e.g. Scillato-Yané et al., 1995; Cione et al., 1999; Carlini \& Scillato-Yané, 1999; Cione \& Tonni, 2005). Hasta el momento su distribución geográfica está circunscripta primariamente a depósitos ensenadenses en las cercanías de las ciudades de Buenos Aires, La Plata y Mar del Plata. Sin embargo, esta especie se registra en el Pleistoceno de la localidad de Villa Larca, provincia de San Luis (Miño-Boilini \& Carlini, 2009).

Para el Bonaerense-Lujanense ( $c a .0,4$ Ma-8,5 ka AP, Pleistoceno medio-Holoceno temprano; Cione et al., 2007), la única especie es Scelidotherium leptocephalum. Sin embargo, esta especie tuvo, a diferencia de $S$. bravardi, una distribución geográfica mucho más amplia y está particulamente bien representada en la región Pampeana (Buenos Aires y Córdoba) en cantidad y calidad de especímenes preservados. En síntesis, S. leptocephalum se encuentra restringida al Bonaerense ( $c a$. 0,4-130 ka; Pleistoceno medio)-Lujanense de la provincia de Buenos Aires, y más recientemente, restos asignables a esta especie han sido hallados en Formosa (Fm. Río Bermejo) y al Lujanense ( $c a$. 130-8,5 ka; Pleistoceno tardío-Holoceno temprano) de las provincias de Córdoba, Santa Fe, Corrientes, Salta y Formosa. De la provincia de Córdoba, procede su registro más moderno (Cruz et al., 2010), que ha sido fechado con $\mathrm{C}^{14}$ en colágeno en $7550 \pm 60$ años AP. Este registro no solo es el más moderno de un escelidoterino, sino el de un perezoso fuera de la región Pampeana de Buenos Aires. Hasta el momento $S$. leptocephalum estuvo primariamente distribuida en el actual territorio de la Argentina, aunque recientemente Corona \& Perea (2010a,b) citan su presencia en el Pleistoceno tardío de Uruguay. 


\section{AGRADECIMIENTOS}

Los autores agradecen a A. Kramarz y L.E. Cruz (MACN); M. Reguero y L. Pomi (MLP); A. Dondas (MMP); J.L. Aguilar (GCF); T.M. de Bianco y R. Tomassini (MD); S. Aramayo (UNS); J.O. Chiesa y N. Lucero (USL); J. F. y J. Friedich (PVE-F); S. Cornero y F. Solomita (MFCA); A. Tauber (CORD-PZ) por facilitarme el acceso a las colecciones bajo su cargo. A F. Góis por el resumo. A E. Oliveira y un revisor anónimo, quienes arbitraron el trabajo, por sus sugerencias que contribuyeron a mejorarlo sustancialmente.

\section{REFERENCIAS}

Ameghino, F. 1881. La formación pampeana o estudio sobre los terrenos de transporte de la cuenca del Plata. Paris-Buenos Aires, G. Masson-Igon Hermanos, 376 p.

Ameghino, F. 1886. Contribución al conocimiento de los mamíferos fósiles de los terrenos terciarios antiguos del Paraná. Boletín de la Academia Nacional de Ciencias de Córdoba, 9: 5-228.

Aramayo, S.A. 1988. Nuevos restos de Proscelidodon sp. (Edentata, Mylodontidae) del yacimiento de Monte Hermoso (Plioceno inferior a medio) provincial de Buenos Aires, Argentina. Estudio morfológico funcional. In: JORNADAS GEOLÓGICAS BONAERENSES, 2, 1988. Actas, Bahía Blanca, p. 99-107.

Bargo, M.S.; De Iuliis, G. \& Vizcaíno, S.F. 2006. Hypsodonty in Pleistocene ground sloths. Acta Palaeontologica Polonica, 51:53-61.

Bidegain, J.C.; Soibelzon, E.; Prevosti, F.J.; Rico, Y.; Verzi, D.H. \& Tonni, E.P. 2005. Magnetoestratigrafía y bioestratigrafía de las barrancas costeras de Necochea (provincia de Buenos Aires, Argentina). In: CONGRESO GEOLÓGICO ARGENTINO, 16, 2005. Actas, La Plata, p. 239-246.

Bosca y Casanoves, E. 1917. Colección paleontológica J. Rodrigo Botet (Donada a Valencia). Restos pertenecientes al género (Scelidotherium) Owen. Publicaciones de la Asociación Española para el Progreso de las Ciencias, 4:35-57.

Carlini, A.A. \& Scillato-Yané, G.J. 1999. Evolution of Quaternary xenarthrans (Mammalia) of Argentina. Quaternary of South America and Antarctic Peninsula, 12:149-175.

Carlini, A.A. \& Scillato-Yané, G.J. 2004. The oldest Megalonychidae (Tardigrada, Xenarthra) and the phylogenetic relationships of the family. Neues Jahrbuch für Geologie ünd Paläontologie, 233:423-443.

Carlini, A.A.; Zurita, A.E.; Gasparini, G.M. \& Noriega, J.I. 2004. Los mamíferos del Pleistoceno de la Mesopotamia argentina y su relación tanto con aquellos del Centro-Norte de la Argentina, Paraguay, sur de Bolivia, como con los del sur de Brasil y oeste de Uruguay: paleobiogeografía y paleoambientes. In: R.G. Aceñolaza (coord.) Temas de la biodiversidad del litoral fluvial argentino, Tucumán, INSUGEO, p. 83-90 (Miscelánea 12).

Carlini, A.A.; Zurita, A.E. \& Miño-Boilini, A.R. 2008. Reseña paleobiogeográfica de los Xenarthra (Mammalia) del Pleistoceno tardio de la región Mesopotámica (Argentina). Tucumán, INSUGEO, p. 259-270 (Miscelánea 17).

Cartelle, C.; De Iuliis, G. \& Lopes-Ferreira, R. 2009. Systematic revision of tropical Brazilian Scelidotheriine sloths (Xenarthra, Mylodontoidea). Journal of Vertebrate Paleontology, 29:555566. doi:10.1671/039.029.0231

Cione, A.L. \& Tonni, E.P. 1995. Bioestratigrafía y cronología del Cenozoico superior de la región pampeana. In: M.T. Alberdi; G.
Leone \& E.P. Tonni (eds.) Evolución climática y biológica de los últimos cinco millones de años. Un ensayo de correlación con el Mediterráneo occidental, Monografías del Museo Nacional de Ciencias Naturales, Consejo Superior de Investigaciones Científicas, p. 49-74.

Cione, A.L. \& Tonni, E.P. 2005. Bioestratigrafía basada en mamíferos del Cenozoico superior de la provincia de Buenos Aires, Argentina. In: CONGRESO GEOLÓGICO ARGENTINO, 16, 2005. Relatorio, La Plata, p. 183-200.

Cione, A.L.; Tonni, E.P.; Bond, M.; Carlini, A.A.; Pardiñas, U.F.J.; Scillato-Yané, G.J.; Verzi, D. \& Vucetich, M.G. 1999. Occurrence charts of Pleistocene mammals in the Pampean area, eastern Argentina. Quaternary of South America and Antarctic Peninsula, 12:53-61.

Cione, A.L. et al. 2007. Mamíferos continentales del Mioceno tardío a la actualidad en la Argentina: cincuenta años de estudios. Ameghiniana, 11:257-278.

Corona, A. \& Perea, D. 2010a. Confirmación de la presencia de Scelidotherium Owen (Xenarthra, Mylodontidae) en Uruguay (Formación Dolores, Pleistoceno Superior). In: SIMPÓSIO BRASILEIRO DE PALEONTOLOGÍA DE VERTEBRADOS, 7, 2010. Boletim de Resumos, Rio de Janeiro, UNIRIO, p. 84.

Corona, A. \& Perea, D. 2010b. Los Scelidotheriinae (Xenarthra, Mylodontidae) de Uruguay: Primera síntesis. In: CONGRESO URUGUAYO DE ZOOLOGÍA, 1, 2010. Libro de resúmenes, Montevideo, Sociedad Zoologica del Uruguay, p. 84.

Cope, E.D. 1889. The Edentata of North America. American Naturalist, 23:657-664.

Cruz, L.E.; Bargo, M.S.; Tonni, E.P. \& Figini, A.J. 2010. Radiocarbon date on megafauna from the late Pleistoceneearly Holocene of Córdoba province, Argentina: stratigraphic and paleoclimatic significance. Revista Mexicana de Ciencias Geológicas, 27:470-476.

Delsuc, F.; Scally, M.; Madsen, O.; Stanhope, M.J.; de Jong, W.W.; Catzeflis, F.M.; Springer, M.S. \& Douzery, E.J.P. 2002. Molecular phylogeny of living Xenarthrans and the impact of character and taxon sampling on the placental tree rooting. Molecular Biology and Evolution, 19:1656-1671.

Esteban, G.I. 1996. Revisión de los Mylodontinae cuaternarios (Edentata-Tardigrada) de Argentina, Bolivia y Uruguay. Sistemática, filogenia, paleobiología, paleozoogeografía y paleoecología. Universidad Nacional de Tucumán, Ph.D. thesis, $235 \mathrm{p}$.

Ferrero B.S. \& Noriega J.I. 2009. La paleontología de vertebrados en el Cuaternario de la provincia de Entre Ríos (Argentina): estado actual y perspectivas. In: A.M. Ribeiro; S.G. Bauermann \& C.S. Scherer (eds.) Quaternário do RS: integrando conhecimentos, Porto Alegre, Sociedade Brasileira de Paleontologia, p. 207-215 (Monografias 1).

Gaudin, T.J. \& McDonald, H.G. 2008. Morphology based investigations of the phylogenetic relationships among extant and fossil xenarthrans. In: S.F. Vizcaíno \& W.J. Loughry (eds.) The Biology of the Xenarthra, University Press of Florida, p. 24-36.

Gill, T. 1872. Arrangement of the families of mammals with analytical tables. Smithsonian Miscellaneous Collections, 11:1-98.

Goloboff, P.A.; Farris, J.S. \& Nixon, K.C. 2008. TNT, a free program for phylogenetic analysis. Cladistic, 24:774-786. doi: 10.1111/j.1096-0031.2008.00217.x

Hoffstetter, R.J. 1952. Les mammifères pleistocenes de la Republique de L'Equateur. Paris, Sociéte géologique de France, p. 1-391 (Mémoire 66). 
International Nommission on Zoological Nomenclature (ICZN). 1999. International Code of Zoological Nomenclature adopted by the XX General Assembly of the International Union of Biological Sciences. London, International Trust for Zoological Nomenclature, p. 1-306.

Kraglievich, L. 1923. Descripción comparada de los cráneos de Scelidodon rothi Ameghino y Scelidotherium parodi n. sp. procedentes del horizonte "chapadmalense". Anales del Museo Nacional de Historia Natural "Bernardino Rivadavia”, 33:57-103.

Kraglievich, L. 1934. La antigüedad Pliocena de las faunas de Monte Hermoso y Chapadmalal, deducidas de su comparación con las que le precedieron y sucedieron. In: A.J. Torcelli (ed.) Obras de Geología y Paleontología III, p. 293-433.

Kraglievich, J.L. 1945. Sobre una mandíbula de Scelidotherium chapadmalense Amegh., procedente del Pampeano medio de Miramar. Anales de la Sociedad Cientifica Argentina, 139:152-162.

Krmpotic, M.C. \& Scillato-Yané, G.J. 2007. Rectificación de la procedencia estratigráfica de Eutatus seguini Gervais, 1867 (Xenarthra, Dasypodidae). Ameghiniana, 44:637-638.

Latham, J., \& Davies, H. 1795. Faunula indica. Appendix. In: J.R. Forster (ed.) Zoologia Indica, Halle A. S., 38 p.

Lydekker, R. 1886. Description of three species of Scelidotherium. Proceedings of the Zoological Society of London, 32:491-498.

Lydekker, R. 1895. Contribution to a knowledge of the fossil Vertebrates of Argentina II: the extinct Edentates of Argentina. Anales del Museo La Plata, 32:1-118.

McDonald, H.G. 1987. A Systematic Review of the Plio-Pleistocene Scelidotherinae Group Sloth (Mammalia: Xenarthra: Mylodontidae). University of Toronto, Ph.D. thesis, $478 \mathrm{p}$.

McDonald, H.G. 2003. Xenarthran skeletal anatomy: primitive or derived? (Mammalia, Xenarthra). Senckenbergiana biologica, 83:5-17.

McDonald, H.G. \& De Iuliis, G. 2008. Fossil history of sloths. In: S.F. Vizcaíno \& W.J. Loughry (eds.) The Biology of the Xenarthra, University Press of Florida, p. 39-55.

McDonald, H.G. \& De Muizon, C. 2002. The cranial anatomy of Thalassocnus (Xenarthra, Mammalia), a derived Nothrothere from the Neogene of the Pisco Formation (Peru). Journal of Vertebrate Paleontology, 22:349-365. doi:10.1671/02724634(2002)022[0349:TCAOTX]2.0.CO;2.

McDonald, H.G. \& Perea, D. 2002. The large Scelidothere Catonyx tarijensis (Xenarthra, Mylodontidae) from the Pleistocene of Uruguay. Journal of Vertebrate Paleontology, 22:677-683. doi:10.1671/0272 4634(2002)022[0677:TLSCTX]2.0.CO;2

McKenna, M.C. \& Bell, S.K. 1997. Classification of mammals above the species level. New York, Columbia University Press, $631 \mathrm{p}$.

Miño-Boilini,A.R. 2012a. Sistemática y evolución de los Scelidotheriinae (Xenarthra, Mylodontidae) cuaternarios de la Argentina. Importancia bioestratigráfica, paleobiogeográfica y paleoambiental. Universidad Nacional de La Plata, Ph.D. thesis, 317 p.

Miño-Boilini, A.R. 2012b. Sistemática y evolución de los Scelidotheriinae (Xenarthra, Mylodontidae) cuaternarios de la Argentina. Importancia bioestratigráfica, paleobiogeográfica y paleoambiental. Mastozoología Neotropical, 19:377-378.

Miño-Boilini, A.R. \& Carlini, A.A. 2009. The Scelidotheriinae Ameghino, 1904 (Phyllophaga, Xenarthra) from the EnsenadanLujanian Stage/Ages (Early Pleistocene to Early-Middle Pleistocene-Early Holocene) of Argentina. Quaternary International, 210:93-101. doi:10.1016/j.quaint.2009.06.016

Miño-Boilini, A.R.; Carlini, A.A.; Chiesa, J.O.; Lucero, N.P. \& Zurita, A.E. 2009. First record of Scelidodon chiliense (Lydekker) (Phyllophaga, Scelidotheriinae) from the Lujanian stage (late Pleistocene-early Holocene) of Argentina. Neues Jahrbuch für Geologie und Paläontologie-Abhandlungen, 253:373-381. doi: 10.1127/0077-7749/2009/0253-0373

Mones, A. 1985. Sobre las fechas de publicación de "Fossil Mammalia" (1839-1840) de Richard Owen. Comunicaciones Paleontológicas del Museo de Historia Natural de Montevideo, 12:199-200.

Mones, A. 1986. Paleovertebrata Sudamericana. Catálogo sistemático de los vertebrados fósiles de América del Sur. Parte I. Lista preliminar y bibliografía. Courier Forschungsinstitut Senckenberg, 82:1-625.

Moreno, F.P. \& Mercerat, A. 1891. Exploración arqueológica de la provincia de Catamarca: Paleontología. Revista del Museo de La Plata, 1:222-236.

Owen, R. 1839. Fossil Mammalia (3). In: C. Darwin (ed.). The Zoology of the Voyage of H.M.S. Beagle, Smith Elder and Co., p. $65-80$.

Owen, R. 1842. Description of the skeleton of an extinct gigantic sloth, Mylodon robustus Owen, with observations on the osteology, natural affinities and probable habits of the megatherioid quadrupeds in general. London, Royal College of Surgeons of England, $176 \mathrm{p}$.

Pascual, R.; Ortega, E.J.; Gondar, D.G. \& Tonni, E.P. 1966. Vertebrata. In: A.V. Borello (ed.) Paleontografia Bonaerense, Comisión de Investigaciones Científicas de la Provincia de Buenos Aires, 202 p.

Paula Couto, C. 1953. Paleontologia Brasileira. Mamíferos. Rio de Janeiro, Instituto Nacional do Livro, 513 p.

Paula-Couto, C. 1979. Tratado de Paleomastozoologia. Rio de Janeiro, Academia Brasileira de Ciências, 590 p.

Pujos, F. 2000. Scelidodon chiliensis (Mammalia, Xeanrthra) du Pleistocene terminal de "Pampa de los Fósiles" (Nord-Pérou). Quaternaire, 11:197-206.

Rovereto, C. 1914. Los estratos araucanos y sus fósiles. Anales del Museo Nacional de Historia Natural de Buenos Aires, 25:1-247.

Rusconi, C. 1936. Distribución de los vertebrados fósiles del Piso Ensenadense. Boletín de la Academia Nacional de Ciencias en Córdoba, 33:183-215.

Schulthess, B. 1920. Beiträge zur Kenntniss der Xenarthra auf Grund der "Santiago Roth'schen Sammlung" des Zoologischen Museum der Universität Zürich, das Skelett der Hand und des Fusses der Xenarthra. Genève, Societe Paléontologique Suisse, 120 p. (Mémoir 44).

Scillato-Yané, G.J. 1977a. Nuevo Megalonychidae (Edentata, Tardigrada) de Edad Chasiquense (Plioceno temprano) del sur de la provincia de Buenos Aires (Argentina). Su importancia filogenética, bioestratigráfica y paleobiogeográfica. Revista de la Asociación de Ciencias Naturales del Litoral, 8:45-54.

Scillato-Yané, G.J. 1977b. Octomylodontinae: n. subfam. de Mylodontidae (Edentata, Tardigrada). Descripción del cráneo y mandíbula de Octomylodon robertoscagliai n. sp., procedentes de la Fm. Arroyo Chasicó (Edad Chasiquense, Plioceno temprano) del S de la prov. de Bs. As. (Argentina). Algunas consideraciones filogenéticas y sistemáticas sobre los Mylodontoidea. Publicación Museo Municipal de Ciencias Naturales "Lorenzo Scaglia”, 2:123-140.

Scillato-Yané, G.J. 1979. Nuevo Nothrotheriinae (Edentata, Tardigrada) de Edad Chasiquense (Plioceno temprano) del S de la Prov. de Bs. As. (Argentina). Su importancia bioestratigráfica, filogenética y paleoambiental. In: CONGRESO GEOLÓGICO ARGENTINO, 7, 1978. Actas, Neuquén, p. 449-457. 
Scillato-Yané, G.J. 1980. Nuevo Megalonychidae (Edentata, Tardigrada) del "Mesopotamiense" (Mioceno tardío-Plioceno) de la Provincia de Entre Ríos (Argentina). Ameghiniana, 17:193-199.

Scillato-Yané, G.J. 1981. Nuevo Mylodontinae (Edentata, Tardigrada) del "Mesopotamiense" (Mioceno tardío-Plioceno) de la Provincia de Entre Ríos (Argentina). Ameghiniana, 18:29-34.

Scillato-Yané, G.J.; Carlini, A.A.; Vizcaíno, S.F. \& Ortiz-Jaureguizar, E. 1995. Los Xenarthros. In: M.T. Alberdi; G. Leone \& E.P. Tonni (eds.) Evolución biológica y climática de la región Pampeana durante los últimos cinco millones de años. Un ensayo de correlación con el Mediterráneo occidental, Monografías del Museo Nacional de Ciencias Naturales, Consejo Superior de Investigaciones Científicas, p. 183-209.

Shockey, B.J. \& Anaya, F. 2011. Grazing in a new Late Oligocene mylodontid sloth and a mylodontid radiation as a component of the Eocene-Oligocene faunal turnover and the early spread of grasslands/savannas in South America. Journal Mammalian Evolution, 18:101-115. doi: 10.1007/s10914-010-9147-5

Soibelzon, E.; Miño-Boilini, A.R.; Zurita, A.E. \& Krmpotic, M.C. 2010. Los Xenarthra (Mammalia) del Ensenadense (Pleistoceno Inferior a Medio) de la Región Pampeana (Argentina). Revista Mexicana de Ciencias Geológicas, 27:449-469.

Soibelzon, E.; Tonni, E.P. \& Bidegain, J.C. 2008. Cronología, magnetoestratigrafía y caracterización bioestratigráfica del Ensenadense (Pleistoceno inferior-medio) en la ciudad de Buenos Aires. Revista de la Asociación Geológica Argentina, 63:421-429.
Soibelzon, E.; Zurita, A.E. \& Carlini, A.A., 2006. Glyptodon munizi (Mammalia, Cingulata, Glyptodontidae): redescripción y anatomía. Ameghiniana, 43:377-385.

Tonni, E.P. 2009. Las unidades portadoras de vertebrados del Cuaternario de las regiones Mesopotámica y Pampeana Oriental de la Argentina: ensayo de correlación. In: A.M. Ribeiro; S.G. Bauermann \& C.S. Scherer (eds.) Quaternário do RS: integrando conhecimentos, Sociedade Brasileira de Paleontologia, p. 57-66 (Monografias 1).

Woodburne, M.; Cione A.L. \& Tonni, E.P. 2006. Central American Provincialism and the Great American Biotic Interchange. In: O. Carranza-Castañeda \& E.H. Lindsay (eds.) Advances in late Tertiary vertebrate paleontology in Mexico and the Great American Biotic Interchange, Publicación Especial del Instituto de Geología y Centro de Geociencias de la Universidad Nacional Autónoma de México, p. 73-101.

Received in March, 2013; accepted in February, 2014. 